Supporting Information

\title{
Fast "ES-Click" Reaction Involving Furfuryl and Triazolinedione Functionalities toward Designing a Healable Polymethacrylate
}

Prantik Mondal ${ }^{\text {a }}$, Gourhari Jana ${ }^{\text {b }}$, Prasanta Kumar Behera ${ }^{a}$, Pratim K. Chattaraj ${ }^{\text {b,c }}$, Nikhil K.

$$
\text { Singha }{ }^{a *}
$$

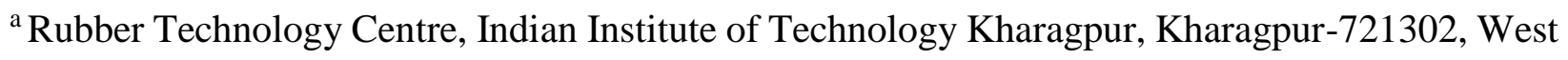
Bengal, India

${ }^{\mathrm{b}}$ Department of Chemistry, Indian Institute of Technology Kharagpur, West Bengal-721302, India

${ }^{c}$ Department of Chemistry, Indian Institute of Technology Bombay, Powai, Mumbai-400076,

India

*nks8888@yahoo.com,nks@rtc.iitkgp.ac.in

Contents of SI:

Pages

1. Experimental Section

1.1 Materials

2

1.2 Characterization Techniques

2-5

1.3 Synthesis of polymers and organic models

Table S1

Figure S1-S9

12-15

Theoretical details

Figure S11-S16

28-31

Table S3

Figure S18

References 


\section{Experimental Section}

\subsection{Materials:}

The monomers, furfuryl methacrylate (FMA), and n-butyl methacrylate (BMA) were obtained from Aldrich, USA. Before polymerization, they were made inhibitor-free by passing through a basic alumina column. $\varepsilon$-caprolactone $(\varepsilon-\mathrm{CL})$, furfuryl amine (FA), stannous octoate $\left(\mathrm{SnOCt}_{2}\right)$, furfuryl alcohol (FOH), 4-cyano-4-[(dodecylsulfanylthiocarbonyl)sulfanyl] pentanoic acid (CDTSPA), 4-Phenyl-1,2,4-triazoline-3,5-dione (PhTAD, 97\%), 4,4'-Methylenebis(phenyl isocyanate), 1,4-Diazabicyclo[2.2.2]octane (DABCO), and 4,4-azobis(4-cyanovaleric acid) (ABCVA) were obtained from Aldrich, USA and used as received. N,N-Dimethylformamide (DMF) and n-hexane solvents were purchased from Merck Specialities Private Limited, Mumbai, India, and used as received. The distillation of THF solvent (obtained from Merck Specialities Private Limited, Mumbai, India) was carried out from the mixture of sodium and benzophenone under the nitrogen atmosphere to make it moisture-free. Bromine (>99\%) was purchased from S. D. Fine Chemical Ltd. and used as received. Ethyl carbazate was purchased from Spectrochem, India, and used as received. 2-Methyl furan (2MF, > $98 \%)$ and trans-2,4-hexadiene-1-ol (HDEO) were bought from Alfa Aesar and used as received.

\subsection{Characterization:}

The Avance III HD $600 \mathrm{MHz}$ (Bruker) spectrometer was used to record the ${ }^{1} \mathrm{H},{ }^{13} \mathrm{C}$, and ${ }^{13} \mathrm{C}$ DEPT135 NMR signals of all polymers and organic compounds. The chemical shifts were referenced in parts per million $(\delta)$ relative to solvent, either $\mathrm{CDCl}_{3}, \mathrm{DMSO}-\mathrm{d}_{6}$ or acetone- $\mathrm{d}_{6}\left(\delta_{\mathrm{H}}=7.27,2.50\right.$, and $2.04 \mathrm{ppm}$ for $\mathrm{CHCl}_{3}, \mathrm{DMSO}$, and acetone, respectively) containing a small amount of tetramethylsilane (TMS) as an internal standard. 
In attenuated total reflection (ATR) mode, within the wavenumber range of $4000-400 \mathrm{~cm}^{-1}$, the FTIR spectra of the polymers were recorded in a Perkin-Elmer (Inc. version 5.0.1) spectrometer. Differential Scanning Calorimetry (DSC) analysis was carried out in a TA Discovery DSC 25 instrument to determine the $\mathrm{T}_{\mathrm{g}}, \mathrm{T}_{\mathrm{m}}$, and retro-ES temperature of the polymers. Under nitrogen atmosphere (with a flow rate of $50 \mathrm{~mL} / \mathrm{min}$ ), the polymethacrylates $(\approx 8 \mathrm{mg}$ ) were scanned (heating-cooling-heating) from $-25{ }^{\circ} \mathrm{C}$ to $+180{ }^{\circ} \mathrm{C}$, and the polycaprolactones $(\approx 8 \mathrm{mg})$ were scanned (heating-cooling-heating) from $-75^{\circ} \mathrm{C}$ to $+180^{\circ} \mathrm{C}$ at a heating rate of $10{ }^{\circ} \mathrm{C} / \mathrm{min}$.

Gel permeation chromatography (GPC) analysis was carried out to determine the average molecular weight and polydispersity index of all the synthesized polymers. Equipped with a refractive index indicator, a Viscotek GPC instrument (model VE 3580, calibrated using linear poly(methyl methacrylate) (PMMA) of narrow polydispersity index as an internal standard) was used to record the measurements under r.t. As eluent, tetrahydrofuran (THF) was used at a flow rate of $1 \mathrm{~mL} / \mathrm{min}$.

Optical microscope (Leica DMLM, made in Germany) was used to analyze the recovery of scratched polymer surface [prepared via drop-casting method $(50 \mathrm{mg} / \mathrm{mL})$ on the glass slide]. The images were taken at a different time interval.

The nanoindentation experiment was carried out using a TribroIndenter TI 950 (Hysitron Inc., Minneapolis, MN, USA) equipped with a diamond indenter tip of $150 \mathrm{~nm}$ radius. At a fixed load of $50 \mu \mathrm{N}$, the hardness and contact depth values of all the polymethacrylate films [(50 mg/ mL), prepared via a drop-casting method on the glass slide] were conducted in a single automated run under r.t.

Contour GT 3D Optical Microscope, Bruker instrument, an optical surface profilometer (OSP), was used to analyze the extent of recovery of the depth of the scratched polymethacrylate films 
$(50 \mathrm{mg} / \mathrm{mL})$ in vertical scan interferometry (VSI) mode. The healing efficacy $\left(E_{H}\right)$ was calculated using the following formula ${ }^{1}$ :

$$
\left(E_{H} \%\right)=\frac{d_{\text {healed }}-d_{\text {damaged }}}{d_{\text {undamaged }}-d_{\text {damaged }}} \times 100
$$

UV-Vis spectroscopy analysis of the organic compounds $(20 \mathrm{mg} / \mathrm{mL})$ and polymer samples $(50$ $\mathrm{mg} / \mathrm{mL}$ ) were carried out in Perkin-Elmer UV/Vis Spectroscopy Lambda 35, using THF as a solvent.

MALDI-TOF-MS analysis was carried out using the Ultraflextreme mass-spectrometer (Bruker). As a matrix and cationic agent, 2,5-Dihydroxybenzoic acid (DHB) and trifluoroacetic acid (TFA) was used. The THF solution of compound $(\approx 20 \mathrm{mg})$ and DHB were mixed at a 1:1 by volume. The rheology analysis was carried out on an Anton Paar MCR 102. The experiments were carried out in the parallel plate geometry model. The crosslinked polymer samples were placed between the plates (of diameter $25 \mathrm{~mm}$ ) by maintaining the gap of 1-2 $\mathrm{mm}$. The experiments were performed using a normal force of $0.2 \mathrm{~N}$, a frequency of $1 \mathrm{~Hz}$, and a strain of $1 \%$. The temperature sweep experiments were conducted from $25^{\circ} \mathrm{C}$ to $120^{\circ} \mathrm{C}$ at a heating rate of $5^{\circ} \mathrm{C} / \mathrm{min}$. To prevent evaporation of solvent during the analysis, DMSO (B.P. $\left.=189^{\circ} \mathrm{C}\right)$ solvent was used..$^{2-5}$

Polymer formulation for rheological analysis:

$\boldsymbol{P F u}$-bisTAD $D_{1}$ : $0.2 \mathrm{~g}$ PFu (0.37 mmol reactive FMA units, in $0.8 \mathrm{~mL}$ DMSO) and $0.0076 \mathrm{~g}(0.021$ $\mathrm{mmol}$ ), in $0.2 \mathrm{~mL}$ DMSO bisTAD were taken separately and dissolved in DMSO. Following the addition of bisTAD (at r.t.), the gelled polymer was deposited (on the bottom plate) and interspersed between the parallel plates. Before the initiation of the analysis, the excess of the polymer formulation was trimmed at the edge of the plates. 
PF $\boldsymbol{u}$-bisTAD $D_{2}: 0.2 \mathrm{~g}$ PFu (0.37 mmol reactive FMA units, in $0.8 \mathrm{~mL}$ DMSO) and $0.068 \mathrm{~g}(0.19$ mmol in $0.2 \mathrm{~mL}$ DMSO) bisTAD were taken separately and dissolved in DMSO. Following the addition of bisTAD (at r.t.), the gelled polymer was deposited (on the bottom plate) and interspersed between the parallel plates. Before the initiation of the analysis, the excess of the polymer formulation was trimmed at the edge of the plates.

The relative crosslink density (CLD) of respective polymers was calculated according to the following formula (equation S1): 6

$$
C L D\left(\mathrm{~mol} / \mathrm{m}^{3}\right)=\frac{\rho}{M_{c}}=\frac{G^{\prime}}{R T}
$$

where $G^{\prime}$ is the storage modulus, $R$ is the universal gas constant $\left(8.314 \mathrm{~J} \mathrm{~K}^{-1} \mathrm{~mol}^{-1}\right), M_{c}$ is the number-average molar mass of the chain lengths between two crosslinks, $\rho$ is the density of the polymer, $T(\mathrm{~K})$ is the temperature.

\section{Computational Details}

Optimization of all of the structures was performed followed by frequency calculations, using a long-range dispersion corrected highly parameterized density functional theory (DFT) based metaGGA approximate functional, M06- $2 X^{7,8}$ in combination with $6-311+\mathrm{G}(\mathrm{d}, \mathrm{p})$ basis set ${ }^{9,10}$ to study the reaction in the gas phase. A hybrid meta-exchange-correlation containing DFT based functional M06-2X was used as it includes double the amount of the non-local exchange, which is known to be well suited in calculating the non-covalent interactions, main-group thermochemistry, kinetics as well as electronic excitation energies of the Rydberg and valence states. ${ }^{8}$ The transition state (TS) search and subsequent intrinsic reaction coordinate (IRC) calculations were performed to confirm that the located TSs are actually connected to the correct stationary points. Zero-point corrections are taken care of in calculating the Gibbs free energies, thermal enthalpy corrections, and entropy. The GAUSSIAN 16 software package ${ }^{11}$ was used for all of the calculations. 


\subsection{Synthesis of polymers and organic models}

Synthesis of poly (FMA29-co-BMA41) copolymer by RAFT polymerization (PFu)

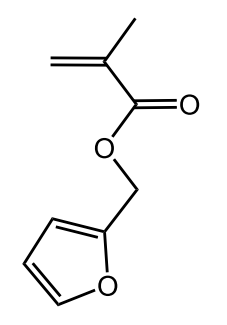

FMA

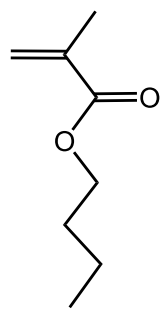

BMA

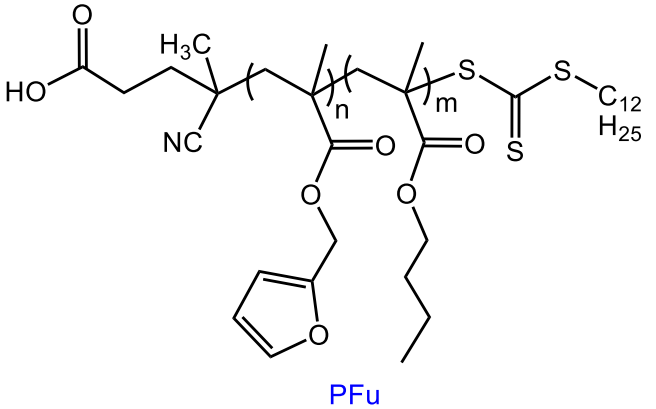

$1 \mathrm{~g}$ of FMA $(0.006 \mathrm{~mol})$ and $1.28 \mathrm{~g}$ of BMA $(0.009 \mathrm{~mol}), 0.0607 \mathrm{~g}$ of CDTSPA $(0.15 \mathrm{mmol}), 0.01$ $\mathrm{g}$ of ABCVA (0.035 mmol) and $2 \mathrm{~mL}$ of DMF solvent were taken in a Schlenk tube enclosed with a silicone rubber septum. The reaction mixture was polymerized at $80{ }^{\circ} \mathrm{C}$ for $16 \mathrm{~h}$ under nitrogen atmosphere. The polymerization was quenched by inserting the tube in ice-bath, resulting in the highly viscous pale-yellow colored polymer. The polymer was solubilized in THF, precipitated from methanol and dried in a vacuum oven at $60{ }^{\circ} \mathrm{C}$ for further analyses.

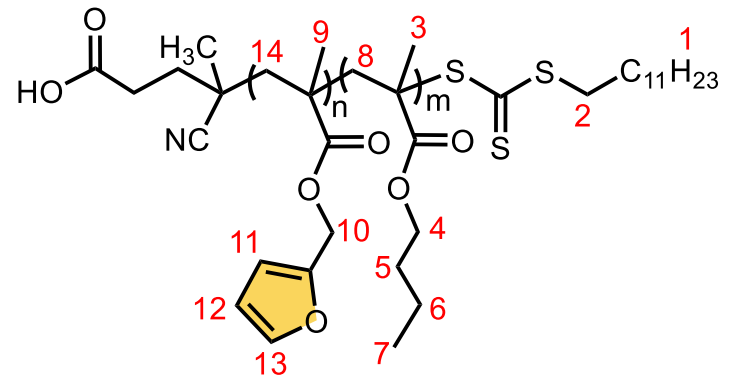

${ }^{1} \mathbf{H}$ NMR (CDCl3, 600 MHz) $\delta$ (ppm): $7.43\left(\mathrm{H}^{13}\right), 6.40-6.35\left(\mathrm{H}^{11,12}\right), 4.97\left(\mathrm{H}^{10}\right), 3.94\left(\mathrm{H}^{4}\right), 3.25$ $\left(\mathrm{H}^{2}\right), 2.20-0.35$ (alkyl Hs).

Synthesis of (4,4'-(4,4'-diphenylmethylene)-bis-(1,2,4-triazoline-3,5-dione)) (bisTAD)

The bisTAD was prepared according to the methodology mentioned in the literature. ${ }^{1}$

Reaction of 2-Methyl furan (2MF) with PhTAD 


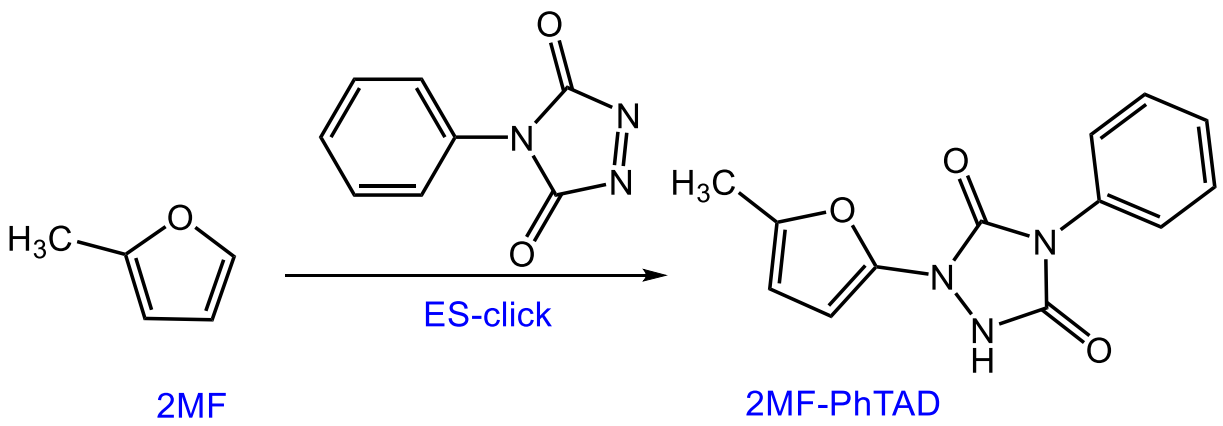

The two organic compounds $2 \mathrm{MF}\left(0.023 \mathrm{~g}, 2.8 \times 10^{-4} \mathrm{~mol}, 0.4 \mathrm{~mL}\right.$ THF solvent $)$ and fresh PhTAD $\left(0.049 \mathrm{~g}, 2.8 \times 10^{-4} \mathrm{~mol}, 0.4 \mathrm{~mL}\right.$ THF solvent) were taken separately and dissolved in $0.5 \mathrm{~mL}$ of THF or $\mathrm{CDCl}_{3}$ (for NMR) solvent. The TAD solution was added to the $2 \mathrm{MF}$ solution under r.t. The completion of modification was confirmed from the rapid discoloration of the azo derivative within less than 10 seconds. The solution was allowed for further spectroscopy analyses.

MALDI-MS $\left[\left(\mathrm{M}+\mathrm{H}^{+}\right)=258.65 \mathrm{Da}, \mathrm{M}=2 \mathrm{MF}-\mathrm{PhTAD}\right.$ adduct); formula weight (F.W) of 2MFPhTAD (F.W. $=257.24)$.

Preparation of polycaprolactone using furfuryl alcohol (FOH) as an initiator $\left(\mathrm{PCL}_{5} \mathrm{Fu}\right)$

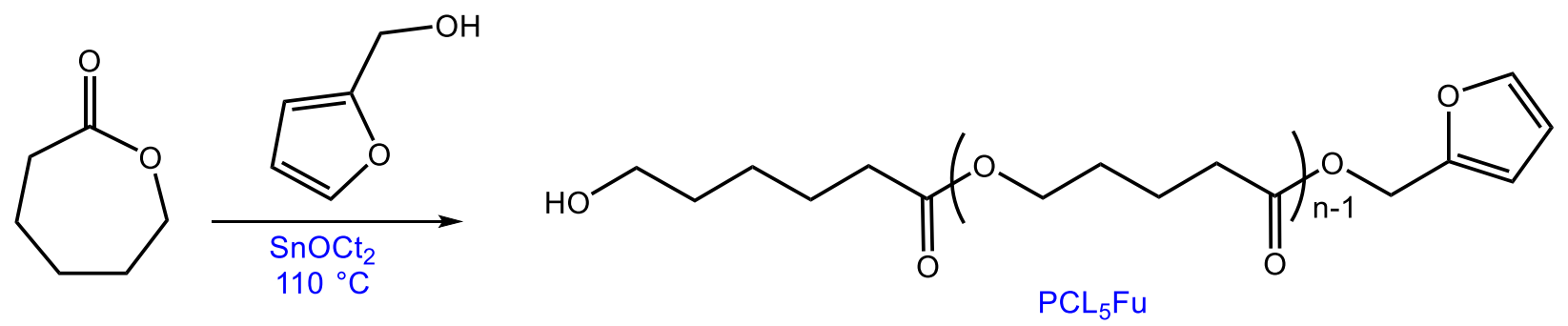

A typical ring-opening polymerization of $\varepsilon$-CL was carried out using $\mathrm{SnOCt}_{2}$ as catalyst and furfuryl alcohol $(\mathrm{FOH})$ as an initiator. The monomer, catalyst, and initiator were taken at an appropriate molar ratio. First, the monomer $(5 \mathrm{~g}, 43 \mathrm{mmol})$ and initiator $(0.86 \mathrm{~g}, 8.7 \mathrm{mmol})$ were taken in a $100 \mathrm{ml}$ round-bottomed flask and allowed to rotate at $140{ }^{\circ} \mathrm{C}$ for $30 \mathrm{~min}$ followed by cooling to $110^{\circ} \mathrm{C}$. Following that, a desired volume of the catalyst was added to the homogeneous reaction mixture and rotated it for $24 \mathrm{~h}$. Finally, the viscous polymer was solubilized in DCM and 
precipitated from hexane. The re-precipitation process was carried out for several times to make the polymer free of excess catalyst and unreacted monomer $\left(\mathrm{M}_{\mathrm{n}, \mathrm{NMR}}=623.14 \mathrm{~g} \mathrm{~mol}^{-1}\right)$.

\section{Conjugation of PCLsFu with PhTAD}

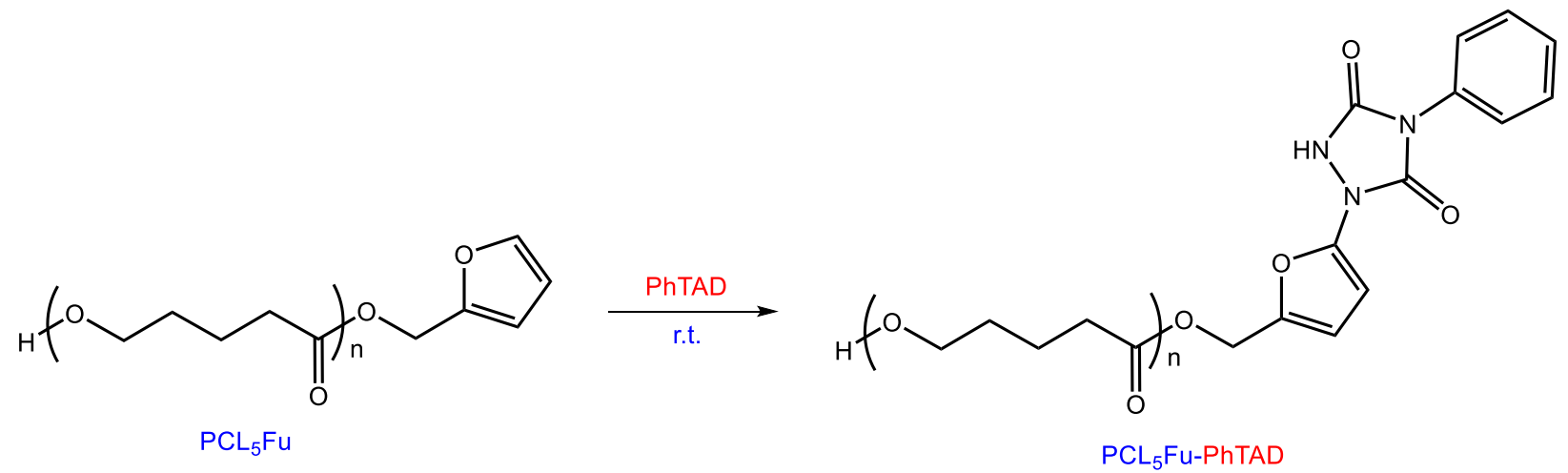

The PCL5Fu polymer (0.1 g, $0.16 \mathrm{mmol}$ terminal groups) and PhTAD (0.028 g, $0.16 \mathrm{mmol})$ were taken and dissolved separately in either $(1 \mathrm{~mL}) \mathrm{THF}$ or $\mathrm{CDCl}_{3}$ solvent. Upon the addition of the TAD solution to the PCLsFu solution, the red color of the azo derivative disappeared immediately. The complete functionalization of the linear polymer bearing reactive furfuryl end group has been further assessed via ${ }^{1} \mathrm{H}$ NMR analysis.

\section{Preparation of polycaprolactone using furfuryl alcohol (FOH) as an initiator (PCL ${ }_{10}$ Fu)}<smiles>CCCCCC(C)C(=O)OCc1ccco1</smiles>

A typical ring-opening polymerization of $\varepsilon$-CL was carried out using $\mathrm{SnOCt}_{2}$ as catalyst and furfuryl alcohol $(\mathrm{FOH})$ as an initiator. The monomer, catalyst, and initiator were taken at an appropriate molar ratio. First, the monomer $(5 \mathrm{~g}, 43 \mathrm{mmol})$ and initiator $(0.43 \mathrm{~g}, 4.3 \mathrm{mmol})$ were 
taken in a $100 \mathrm{ml}$ round-bottomed flask and allowed to rotate at $140{ }^{\circ} \mathrm{C}$ for $30 \mathrm{~min}$ followed by cooling to $110^{\circ} \mathrm{C}$. Following that, a desired volume of the catalyst was added to the homogeneous reaction mixture and allowed to rotate it for $24 \mathrm{~h}$. The viscous polymer was solubilized in DCM and precipitated from hexane. The re-precipitation process was carried out for several times to make the polymer free of excess catalyst and unreacted monomer. $\left(\mathrm{M}_{\mathrm{n}, \mathrm{GPC}}=1200 \mathrm{~g} \mathrm{~mol}^{-1}, \mathrm{Ð}=\right.$ $1.21)$.

\section{Conjugation of PCL $10 F u$ with PhTAD}

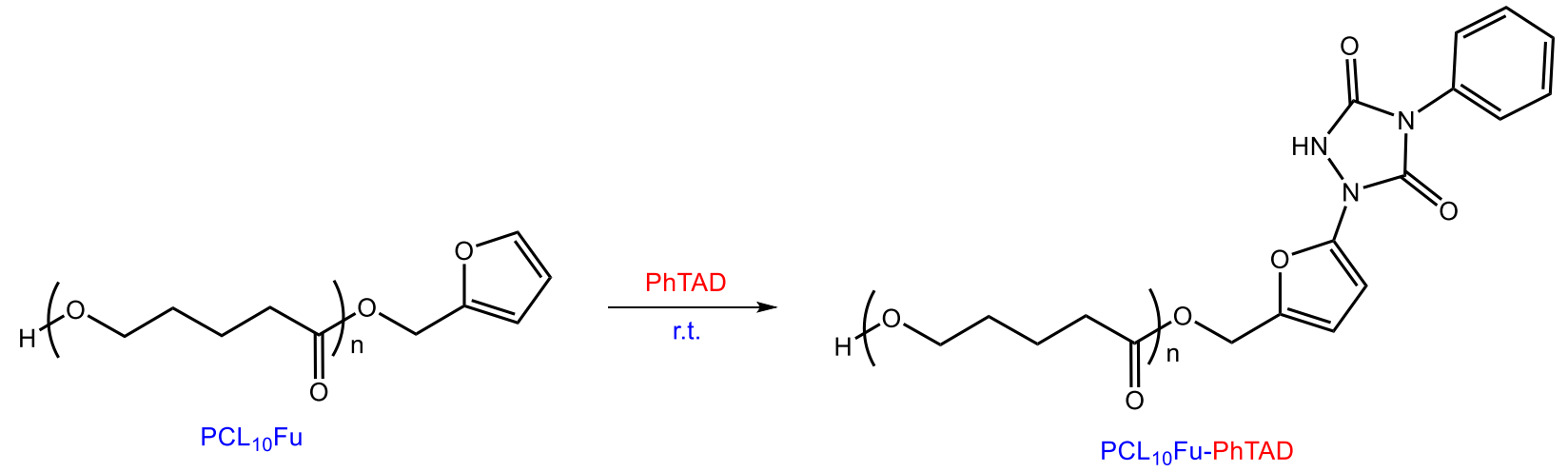

The PCL10Fu polymer (0.1 g, $0.083 \mathrm{mmol}$ terminal groups) and PhTAD (0.013 g, $0.075 \mathrm{mmol})$ were taken and dissolved separately in either $(0.5 \mathrm{~mL}) \mathrm{THF}$ or $\mathrm{CDCl}_{3}$ solvent. Upon the addition of TAD solution to the PCL10Fu solution, the red color of azo derivative disappeared immediately. The complete functionalization of the linear polymer bearing reactive furfuryl end group has been further assessed via GPC and ${ }^{1} \mathrm{H}$ NMR analysis. $\left(\mathbf{M}_{\mathrm{n}, \mathrm{GPC}}\right.$ of PCL10Fu-PhTAD $=1.4 \mathrm{~kg} \mathrm{~mol}^{-1}$, PDI $=1.30)$

\section{Modification of PFu copolymer with Phenyl-TAD (PhTAD) via ES-click chemistry}

In a typical post-polymerization modification reaction, $0.1 \mathrm{~g}(0.18$ mmol reactive FMA units) of PFu and $0.0035 \mathrm{~g}(0.019 \mathrm{mmol})$ PhTAD (molar ratio of FMA: PhTAD = 9:1) were dissolved in $0.5 \mathrm{ml}$ of THF separately. The successful derivation of $\mathbf{P F u}$ was evidenced by the gradual 
disappearance of the red color of the TAD derivative within a few seconds after the addition of PhTAD. The whole reaction was carried out at room temperature (r.t.) $\left(\sim 30^{\circ} \mathrm{C}\right)$ for completion.

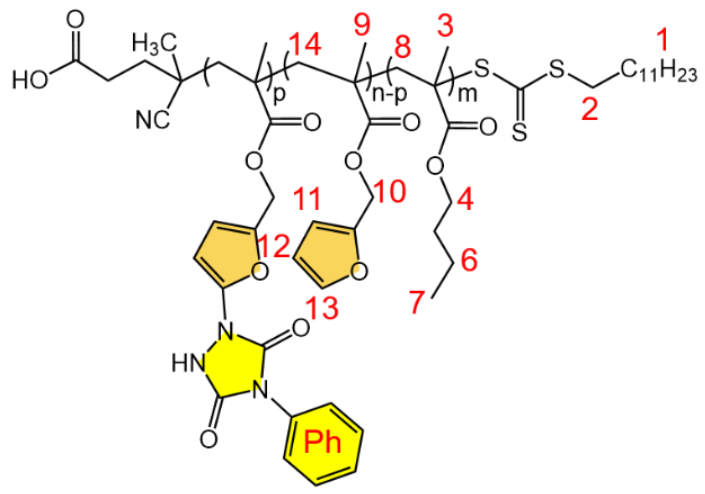

${ }^{1} \mathrm{H}$ NMR (CDCl, 600 MHz) $\boldsymbol{\delta}$ (ppm): 7.55-7.39 $\left(\mathrm{H}^{\mathrm{Ph}}\right), 7.43\left(\mathrm{H}^{13}\right), 6.40-6.35\left(\mathrm{H}^{11,12}\right), 4.97\left(\mathrm{H}^{10}\right)$, $3.94\left(\mathrm{H}^{4}\right), 3.25\left(\mathrm{H}^{2}\right), 2.20-0.35$ (alkyl Hs).

Preparation of chemical network of PFu copolymer with bisTAD via ES-click chemistry

In case of a copolymer, $0.1 \mathrm{~g}$ of PFu $(0.18 \mathrm{mmol}$ reactive FMA units $)$ and $0.0037 \mathrm{~g}(0.01 \mathrm{mmol})$ of bisTAD (molar ratio of FMA: bisTAD = 18:1) were dissolved separately in $0.5 \mathrm{~mL}$ of THF solvent and mixed at r.t. After the addition of the TAD solution, the polymer solution got immediately gelled. As evidenced by the gradual disappearance of the TAD color, the overall modification was completed within 2 min under r.t. The crosslinked polymer was dried in a vacuum oven and stored for further analyses. 
Table S1. Summary of PFu polymer

\begin{tabular}{cccccccc}
\hline Polymer & $\begin{array}{c}\text { Feed } \\
\text { ratio }\end{array}$ & $\begin{array}{c}\text { Monomer } \\
\text { conversion } \\
(\%)\end{array}$ & $\begin{array}{c}\text { Mn, Theo } \\
(\mathbf{g} / \mathbf{m o l})\end{array}$ & $\begin{array}{c}\mathbf{M n}_{\mathbf{n}, \mathbf{N M R}} \\
(\mathbf{g} / \mathbf{m o l})^{\mathrm{b}}\end{array}$ & $\begin{array}{c}\mathbf{M}_{\mathbf{n}, \mathbf{G P C}} \\
(\mathbf{g} / \mathbf{m o l})^{\mathrm{c}}\end{array}$ & $\mathbf{D}^{\mathrm{c}}$ & $\begin{array}{c}\text { Copolymer } \\
\text { composition } \\
(\mathbf{m o l} \%)^{\mathbf{b}}\end{array}$ \\
\hline PFu $^{\text {a }}$ & $60: 40$ & 70 & 12500 & 11100 & 15300 & 1.39 & $59: 41$ \\
\hline
\end{tabular}

${ }^{\mathrm{a}}$ [Monomer]:[CTA]:[initiator] $=100: 1: 0.25$, where the feed ratio of the particular monomers are $[\mathrm{BMA}]:[\mathrm{FMA}]=60: 40$.

${ }^{\mathrm{b}} \mathrm{M}_{\mathrm{n}, \mathrm{NMR}}$ has been calculated from NMR peak intensities.

${ }^{\mathrm{c}} \mathrm{M}_{\mathrm{n}, \text { GPC }}$ and dispersity (Đ) have been calculated from GPC using THF as eluent.

${ }^{\mathrm{d}}$ Copolymer composition was determined from ${ }^{1} \mathrm{H}$ NMR analysis. 


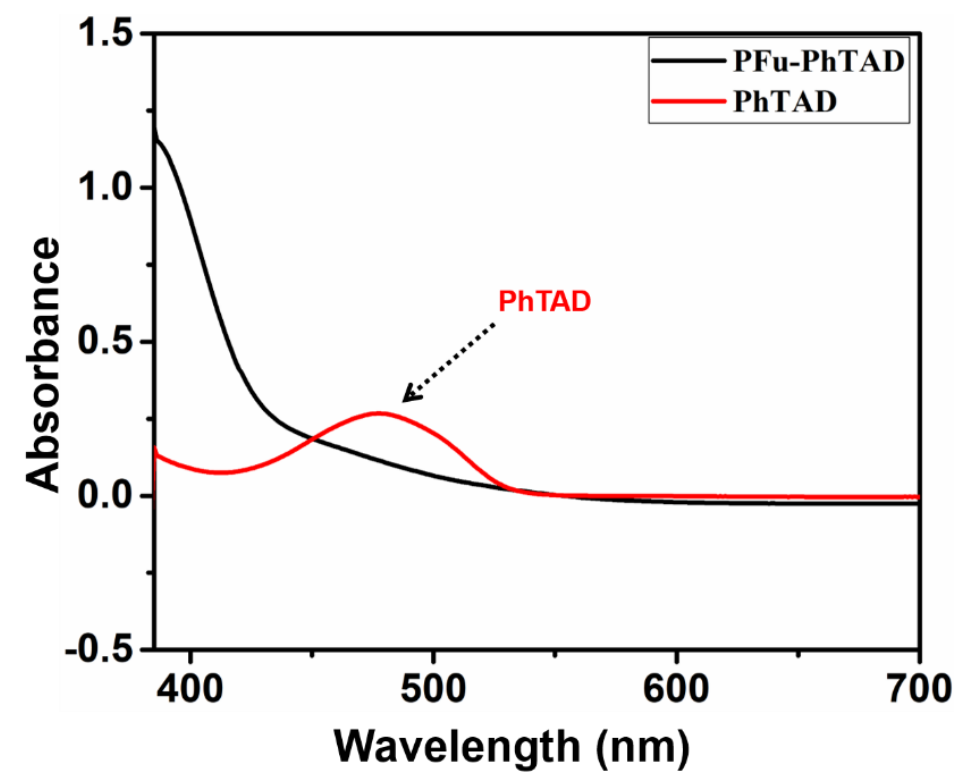

Figure S1. UV analysis of PhTAD and after reaction with PFu polymer (PFu-PhTAD).

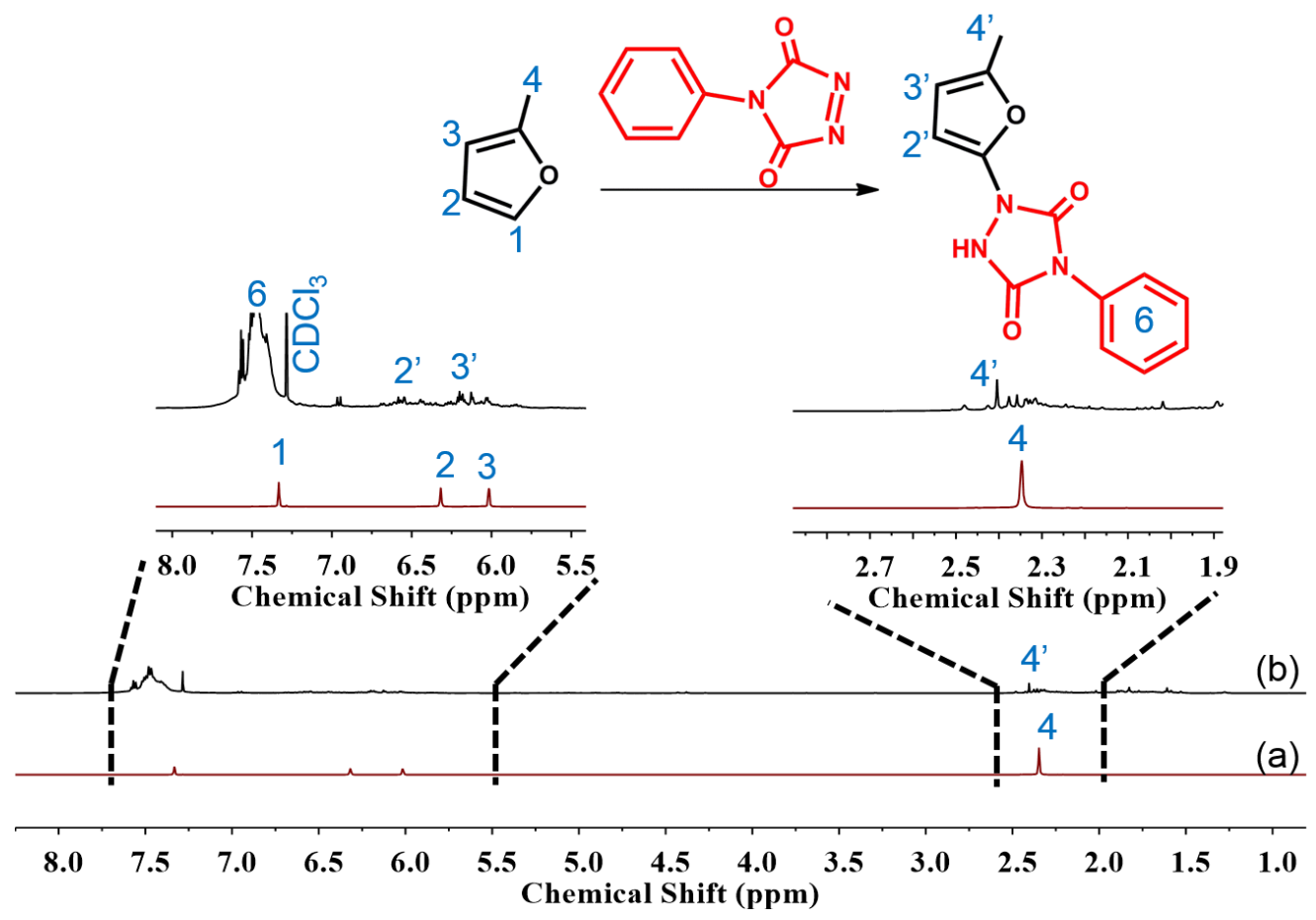

Figure S2. ${ }^{1} \mathrm{H}$ NMR spectroscopy of $2 \mathrm{MF}-\mathrm{PhTAD}(1.1 \mathrm{~mol} \mathrm{PhTAD})$ in $\mathrm{CDCl}_{3}$ solvent. 


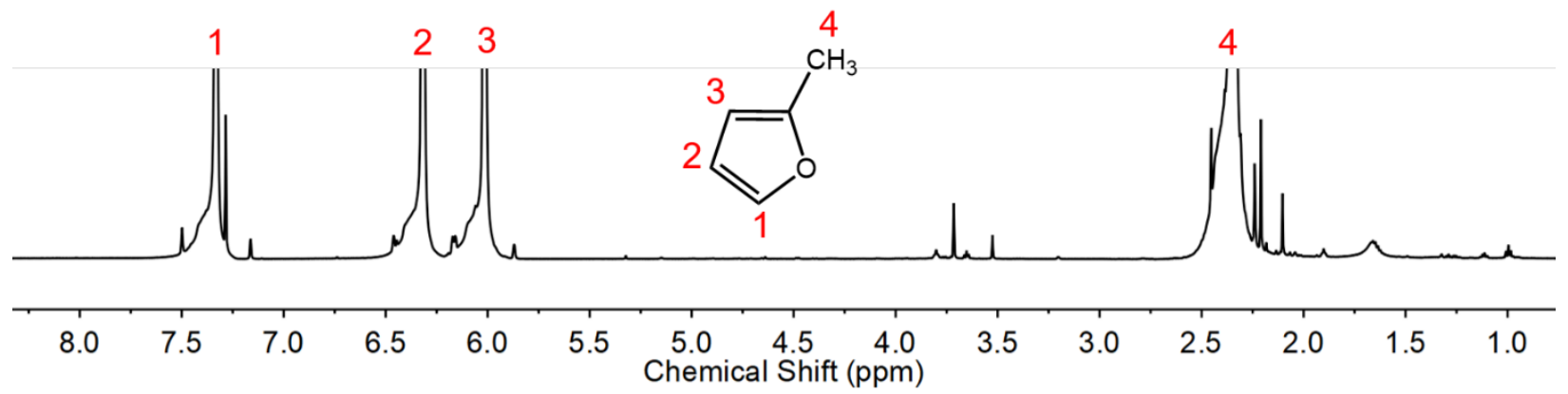

Figure S3. Amplified ${ }^{1} \mathrm{H}$ NMR spectrum of $2 \mathrm{MF}$.

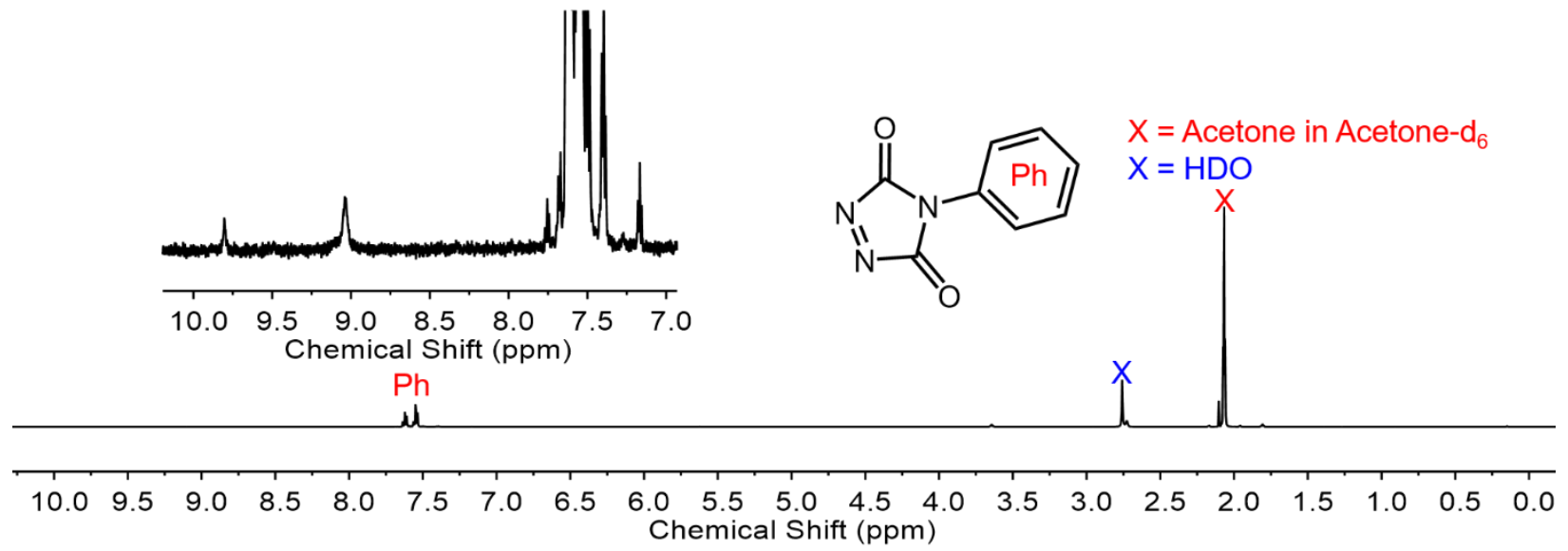

Figure S4. ${ }^{1} \mathrm{H}$ NMR spectrum of PhTAD in acetone $-\mathrm{d}_{6}$.

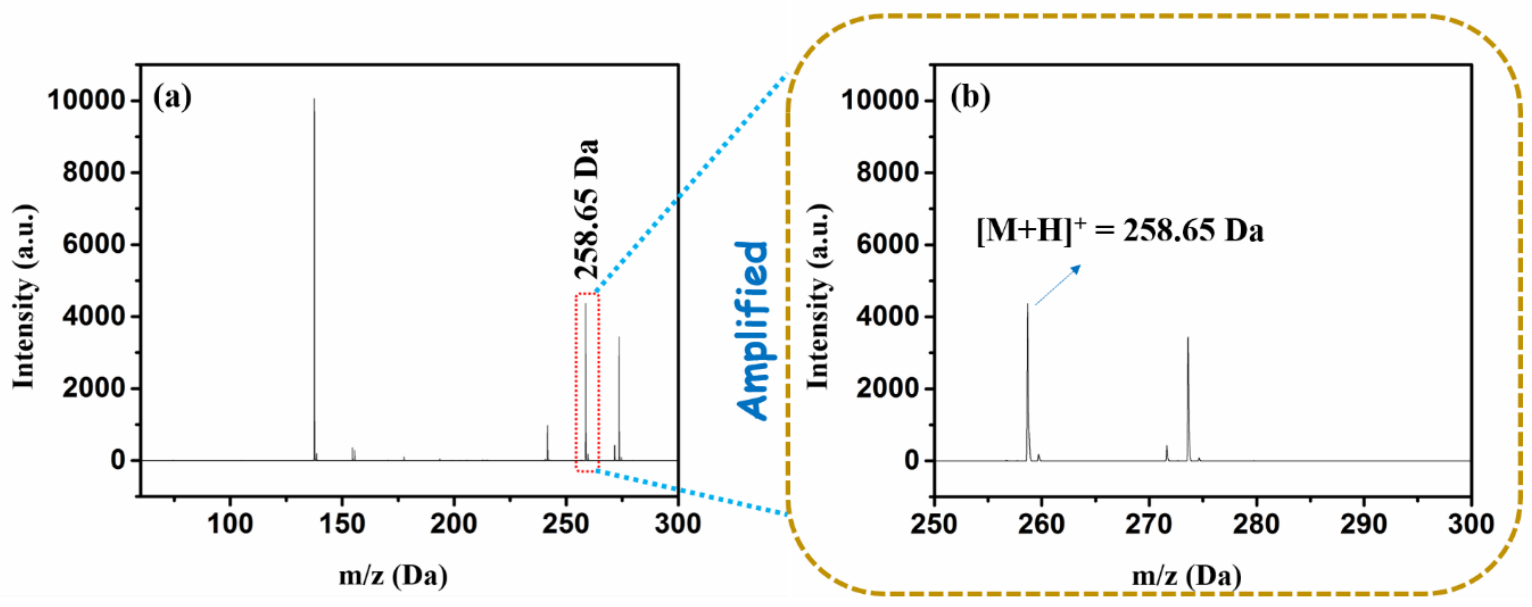

Figure S5. MALDI-MS analysis of 2MF-PhTAD ES-click adduct. 


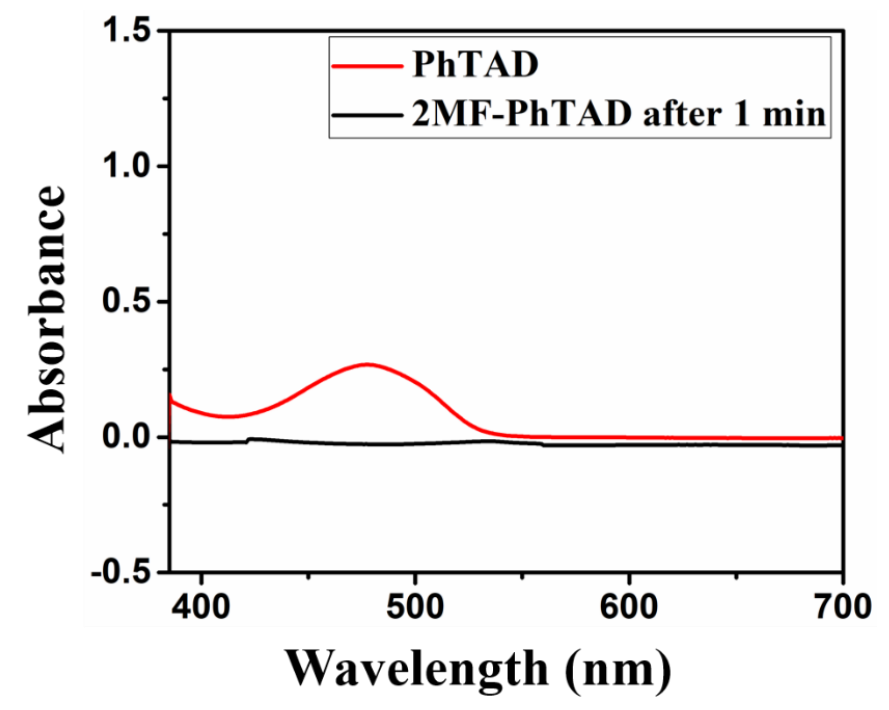

Figure S6. UV-Vis spectroscopy analysis of 2MF-PhTAD.

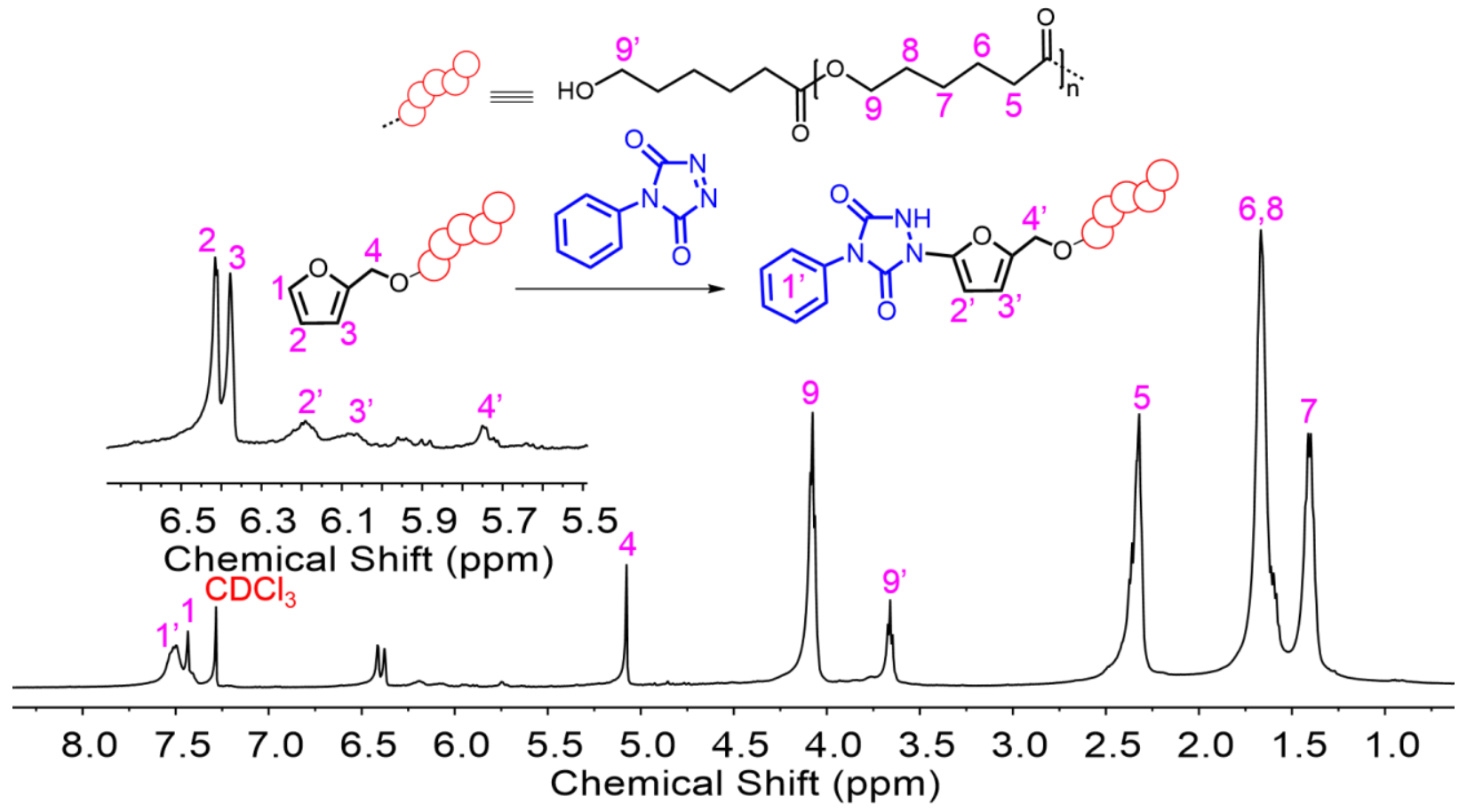

Figure S7. ${ }^{1} \mathrm{H}$ NMR analysis of PhTAD derived PCLsFu $(0.080 \mathrm{mmol}$ PhTAD was added to $0.16 \mathrm{mmol}^{\mathbf{P C L} 5 \mathbf{F u}}$ in $\left.2 \mathrm{~mL} \mathrm{CDCl}_{3}\right)$. 


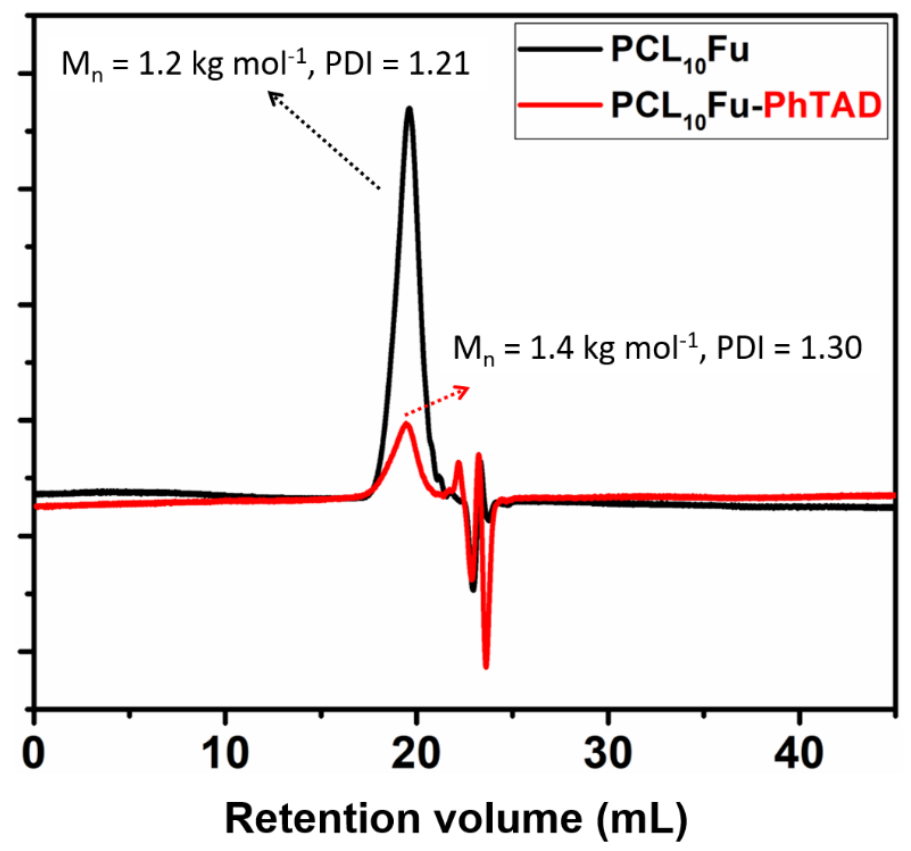

Figure S8. GPC traces of PCL10Fu and PCL10Fu-PhTAD

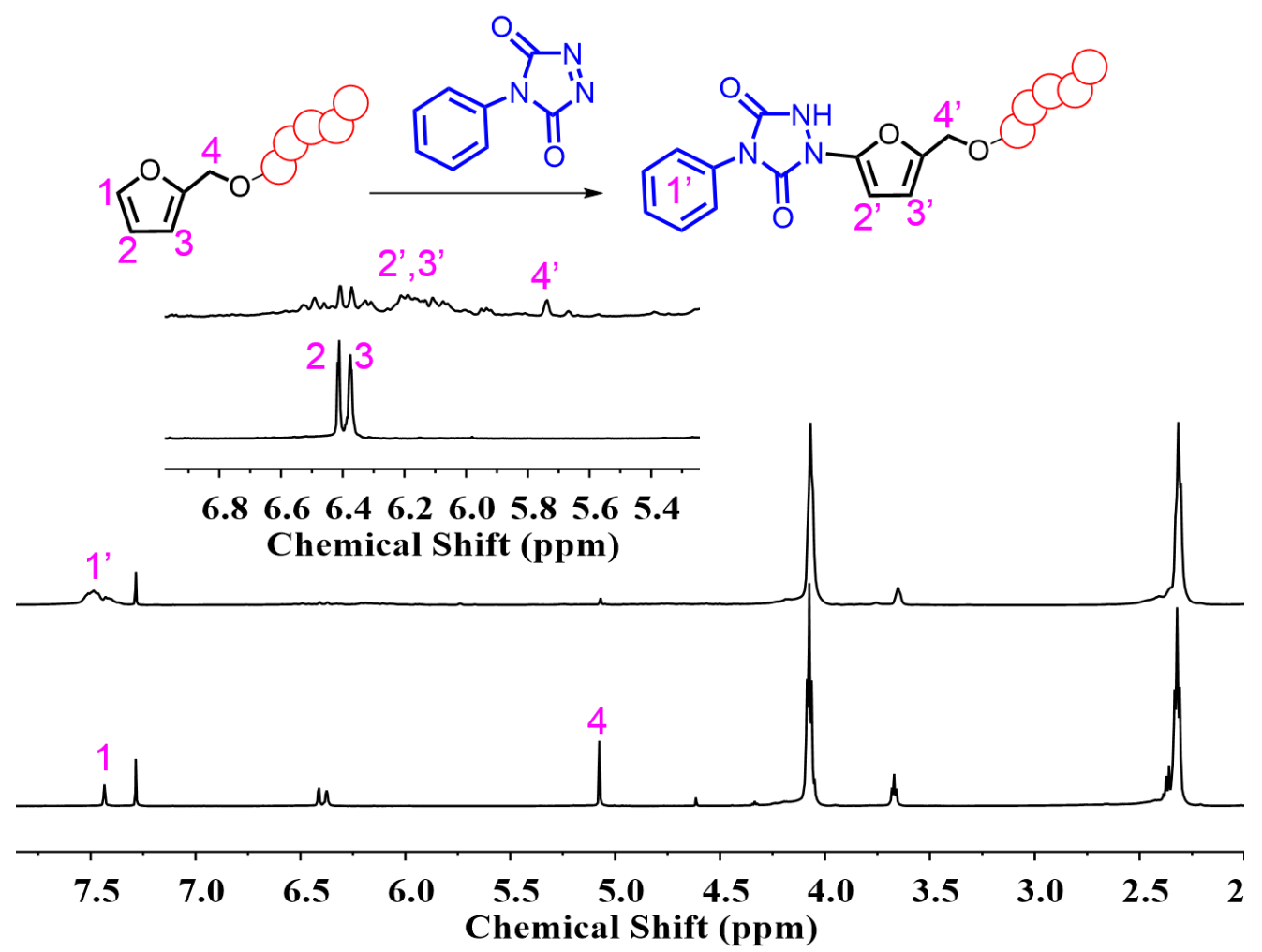

Figure S9. ${ }^{1} \mathrm{H}$ NMR analysis of PCL10Fu-PhTAD in $\mathrm{CDCl}_{3}$. 
Table S2. Summary of change of relative free energy $(\Delta G)$ of 2MF-PhTAD with temperature $(T)$

\begin{tabular}{ccc}
\hline $\boldsymbol{T}(\mathbf{K})$ & $\boldsymbol{T}\left({ }^{\circ} \mathbf{C}\right)$ & $\Delta G(\mathbf{k c a l} / \mathbf{m o l})$ \\
\hline 273 & 0 & -26.5 \\
\hline 298 & 25 & -25.5 \\
\hline 373 & 100 & -22.2 \\
\hline 773 & 500 & -5.1 \\
\hline 873 & 600 & -1.0 \\
\hline 890 & 617 & -0.3 \\
\hline 895 & 622 & -0.1 \\
\hline 896 & 623 & 0.0 \\
\hline 898 & 625 & 0.1 \\
\hline 900 & 627 & 0.1 \\
\hline
\end{tabular}




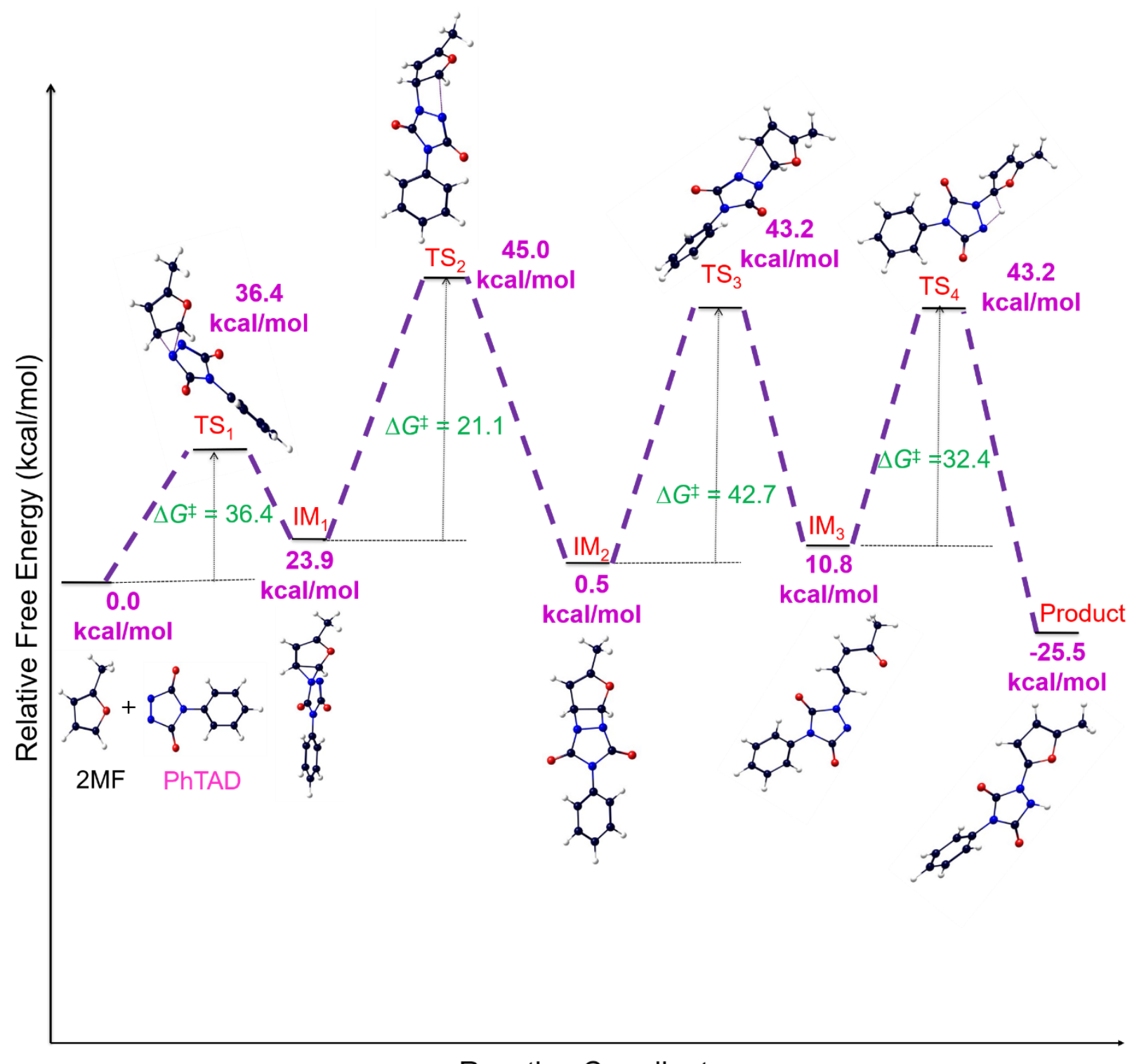

Reaction Coordinate

Figure S10. The minimum energy structures of reactants, intermediates, product and the corresponding transition states obtained at the M06-2X/6-311+G(d,p) level of Path-ES 2. 


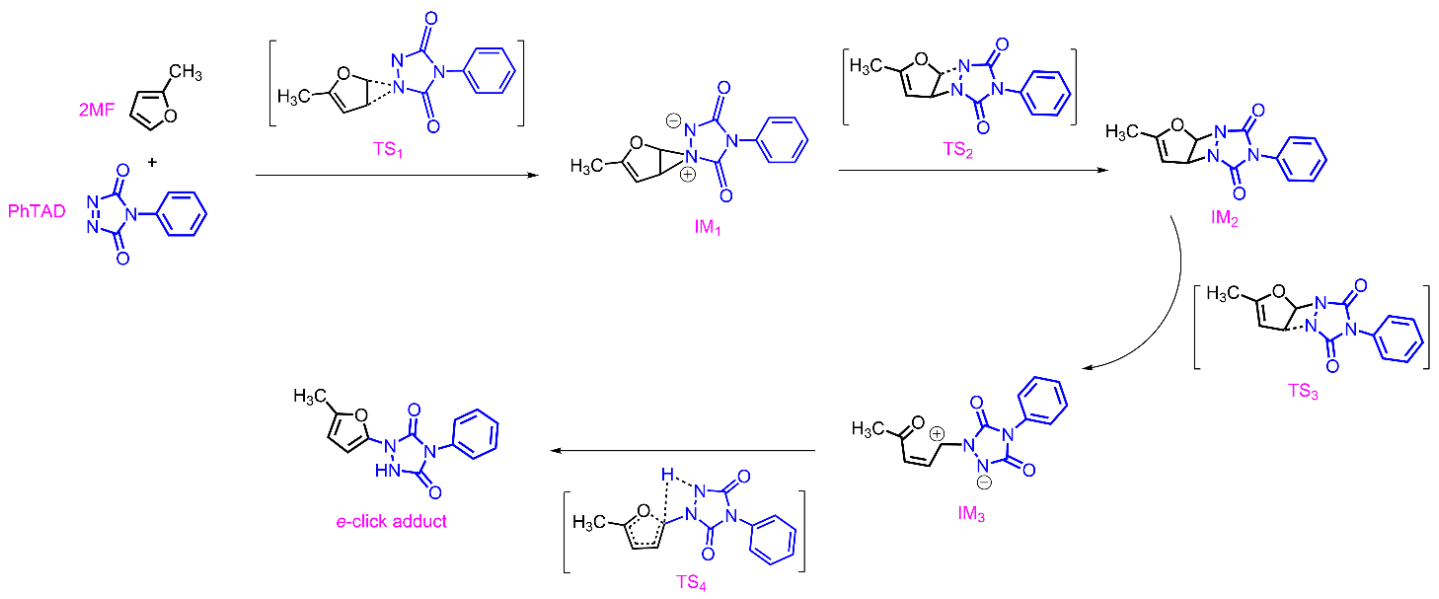

Scheme S1. Schematic illustration of ES-click conjugation of PhTAD with 2MF [designation of the transition states (TSs) and intermediates (IMs) was kept the same as per the Figure S10].

\section{Reaction coordinates for Path-ES 1}

Coordinate of $2 M F$

$\begin{array}{lrrr}\mathrm{C} & 0.63451500 & 0.10590400 & 0.00000600 \\ \mathrm{C} & -0.21874000 & 1.16153600 & 0.00000500 \\ \mathrm{C} & -1.54537500 & 0.61151300 & -0.00014400 \\ \mathrm{C} & -1.38926300 & -0.73240300 & 0.00008600 \\ \mathrm{O} & -0.07111800 & -1.05414800 & 0.00001500 \\ \mathrm{H} & 0.06147300 & 2.20257700 & 0.00000100 \\ \mathrm{H} & -2.48036800 & 1.14706800 & -0.00026100 \\ \mathrm{H} & -2.07773200 & -1.55999300 & 0.00015200 \\ \mathrm{C} & 2.11527400 & -0.01628300 & 0.00002700 \\ \mathrm{H} & 2.46101100 & -0.55701900 & 0.88409400 \\ \mathrm{H} & 2.46103400 & -0.55707900 & -0.88399400 \\ \mathrm{H} & 2.56506000 & 0.97602900 & 0.00000000\end{array}$

Coordinate of PhTAD

$\begin{array}{lrrr}\mathrm{C} & 1.56924400 & 1.09597100 & 0.24685500 \\ \mathrm{C} & 1.56921600 & -1.09573200 & -0.24781900 \\ \mathrm{~N} & 0.76011300 & 0.00009200 & -0.00031600 \\ \mathrm{~N} & 2.97785400 & 0.59785400 & 0.13660900 \\ \mathrm{~N} & 2.97783900 & -0.59803500 & -0.13586100 \\ \mathrm{O} & 1.28209600 & 2.21688800 & 0.49569500 \\ \mathrm{O} & 1.28203100 & -2.21688300 & -0.49556000 \\ \mathrm{C} & -0.66859100 & 0.00004800 & -0.00012000 \\ \mathrm{C} & -1.34952000 & -1.07231400 & 0.56575200\end{array}$




$\begin{array}{lrrr}\mathrm{C} & -1.34974600 & 1.07234700 & -0.56584000 \\ \mathrm{C} & -2.73869000 & -1.06763300 & 0.55663700 \\ \mathrm{H} & -0.80146200 & -1.89932100 & 0.99774500 \\ \mathrm{C} & -2.73891300 & 1.06753000 & -0.55641200 \\ \mathrm{H} & -0.80186600 & 1.89940200 & -0.99796500 \\ \mathrm{C} & -3.43490500 & -0.00008300 & 0.00019300 \\ \mathrm{H} & -3.27532000 & -1.90142900 & 0.99213200 \\ \mathrm{H} & -3.27571900 & 1.90127200 & -0.99179200 \\ \mathrm{H} & -4.51785800 & -0.00013300 & 0.00031600\end{array}$

Coordinate of $\mathrm{TS}_{1}$

$\begin{array}{lrrr}\mathrm{C} & -2.26687100 & -0.32108400 & 0.86338900 \\ \mathrm{C} & -2.64187900 & -1.62963100 & 0.40668100 \\ \mathrm{C} & -3.81259600 & -1.47818300 & -0.30412800 \\ \mathrm{C} & -4.20958200 & -0.14769200 & -0.13060400 \\ \mathrm{O} & -3.37709000 & 0.50010600 & 0.65695000 \\ \mathrm{H} & -2.03033700 & -2.51102300 & 0.50366000 \\ \mathrm{H} & -4.34505600 & -2.21523500 & -0.88326200 \\ \mathrm{C} & 0.50782200 & 1.66683100 & 0.22880000 \\ \mathrm{C} & 0.00177400 & -0.48094400 & -0.31778500 \\ \mathrm{~N} & 1.04191400 & 0.35717600 & -0.07449900 \\ \mathrm{~N} & -1.19119800 & 0.37491500 & -0.28856700 \\ \mathrm{O} & -0.00991600 & -1.66527300 & -0.54192500 \\ \mathrm{O} & 1.19034800 & 2.61731200 & 0.50510500 \\ \mathrm{~N} & -0.85405100 & 1.61143000 & 0.06376300 \\ \mathrm{H} & -1.78484500 & -0.13788800 & 1.81490200 \\ \mathrm{C} & 2.41695200 & -0.00151700 & -0.06401000 \\ \mathrm{C} & 3.36393600 & 0.91104800 & -0.52531900 \\ \mathrm{C} & 2.80731600 & -1.25718100 & 0.39712400 \\ \mathrm{C} & 4.70768600 & 0.55960700 & -0.51535700 \\ \mathrm{H} & 3.04636000 & 1.88602900 & -0.86753800 \\ \mathrm{C} & 4.15453100 & -1.59804100 & 0.39038400 \\ \mathrm{H} & 2.06240900 & -1.96122100 & 0.74156200 \\ \mathrm{C} & 5.10827700 & -0.69299700 & -0.06180500 \\ \mathrm{H} & 5.44415200 & 1.27132400 & -0.86830200 \\ \mathrm{H} & 4.45684300 & -2.57642400 & 0.74394900 \\ \mathrm{H} & 6.15757600 & -0.96200000 & -0.06107300 \\ \mathrm{C} & -5.37515000 & 0.59975900 & -0.64635500 \\ \mathrm{H} & -5.92143500 & 1.05196200 & 0.18460300 \\ \mathrm{H} & -5.02705400 & 1.41005700 & -1.29214100 \\ \mathrm{H} & -6.03529700 & -0.05723500 & -1.20838000\end{array}$

$\underline{\text { Coordinate of } \mathrm{IM}_{1}}$ 


$\begin{array}{lrrr}\mathrm{C} & -2.10651900 & 0.37457500 & 0.03073500 \\ \mathrm{C} & -2.68232500 & -0.93415600 & -0.14799900 \\ \mathrm{C} & -4.00903900 & -1.17218700 & -0.18400200 \\ \mathrm{C} & -5.11155200 & -0.17414500 & -0.04485600 \\ \mathrm{O} & -4.90933900 & 1.00493400 & 0.13753200 \\ \mathrm{H} & -2.00815200 & -1.77032800 & -0.26416700 \\ \mathrm{H} & -4.32500000 & -2.20020300 & -0.33094000 \\ \mathrm{C} & 0.99854000 & 1.78995500 & 0.23151400 \\ \mathrm{C} & 0.28580400 & -0.38044900 & -0.03716300 \\ \mathrm{~N} & 1.37892300 & 0.38960500 & 0.05182400 \\ \mathrm{~N} & -0.82404200 & 0.62883500 & 0.07694200 \\ \mathrm{O} & 0.12751500 & -1.55701000 & -0.17026200 \\ \mathrm{O} & 1.80847200 & 2.66443200 & 0.35060100 \\ \mathrm{~N} & -0.35808800 & 1.87187100 & 0.23371400 \\ \mathrm{H} & -2.73719400 & 1.24800500 & 0.13450600 \\ \mathrm{C} & 2.71703700 & -0.09890700 & 0.00096400 \\ \mathrm{C} & 3.67654900 & 0.61670200 & -0.70885200 \\ \mathrm{C} & 3.04175700 & -1.28239400 & 0.65701800 \\ \mathrm{C} & 4.97854400 & 0.13444800 & -0.75498600 \\ \mathrm{H} & 3.40613100 & 1.54152600 & -1.19958300 \\ \mathrm{C} & 4.34557100 & -1.75746200 & 0.59263500 \\ \mathrm{H} & 2.28100600 & -1.82627400 & 1.20173700 \\ \mathrm{C} & 5.31561000 & -1.05102300 & -0.11015300 \\ \mathrm{H} & 5.73151600 & 0.68959800 & -1.30085600 \\ \mathrm{H} & 4.60184900 & -2.67992300 & 1.09898000 \\ \mathrm{H} & 6.33213300 & -1.42253000 & -0.15411100 \\ \mathrm{C} & -6.50671200 & -0.73878800 & -0.14796300 \\ \mathrm{H} & -7.23618500 & 0.05819700 & -0.02470100 \\ \mathrm{H} & -6.64053900 & -1.22110700 & -1.12021300 \\ \mathrm{H} & -6.65589800 & -1.50499700 & 0.61766800\end{array}$

$\underline{\text { Coordinate of } \mathrm{TS}_{2}}$

$\begin{array}{lrrr}\mathrm{C} & -2.23275200 & 0.21521300 & 0.12696500 \\ \mathrm{C} & -2.47323900 & -0.88160500 & 1.00895600 \\ \mathrm{C} & -3.81531700 & -1.18310500 & 0.95259800 \\ \mathrm{C} & -4.35772600 & -0.35344000 & -0.03727700 \\ \mathrm{O} & -3.44934900 & 0.41334200 & -0.57511200 \\ \mathrm{H} & -1.71091300 & -1.32238600 & 1.63531300 \\ \mathrm{H} & -4.36652400 & -1.90308100 & 1.53526100 \\ \mathrm{C} & 0.63491100 & 1.69458000 & 0.31080400 \\ \mathrm{C} & 0.01153300 & -0.38552800 & -0.51392500 \\ \mathrm{~N} & 1.08191600 & 0.38024300 & -0.12804600 \\ \mathrm{~N} & -1.06071000 & 0.52003700 & -0.57149800 \\ \mathrm{O} & -0.07250700 & -1.57177900 & -0.73133200\end{array}$




$\begin{array}{lrrr}\mathrm{O} & 1.36354700 & 2.49953800 & 0.83741700 \\ \mathrm{~N} & -0.68656500 & 1.79537600 & 0.01439000 \\ \mathrm{H} & -1.96097200 & 1.28557900 & 0.78287600 \\ \mathrm{C} & 2.43096800 & -0.06571100 & -0.07946400 \\ \mathrm{C} & 3.45468600 & 0.84185900 & -0.34993700 \\ \mathrm{C} & 2.72600400 & -1.39348800 & 0.22707800 \\ \mathrm{C} & 4.77468200 & 0.41395400 & -0.30612000 \\ \mathrm{H} & 3.21208000 & 1.87200500 & -0.57062500 \\ \mathrm{C} & 4.05278900 & -1.80810400 & 0.25669500 \\ \mathrm{H} & 1.92532300 & -2.09348400 & 0.41943900 \\ \mathrm{C} & 5.08038700 & -0.90976800 & -0.00570100 \\ \mathrm{H} & 5.56813600 & 1.12269800 & -0.51086200 \\ \mathrm{H} & 4.27954600 & -2.84201600 & 0.48893000 \\ \mathrm{H} & 6.11237300 & -1.23836600 & 0.02255100 \\ \mathrm{C} & -5.75639900 & -0.19436400 & -0.49499200 \\ \mathrm{H} & -6.36853500 & -1.02242600 & -0.14300500 \\ \mathrm{H} & -6.16289800 & 0.74156800 & -0.10166600 \\ \mathrm{H} & -5.78679600 & -0.14144400 & -1.58400000\end{array}$

Coordinate of Product

$\begin{array}{lrrr}\mathrm{C} & 2.31667700 & -0.35650800 & 0.00380300 \\ \mathrm{C} & 2.74099200 & -1.51247600 & 0.56152500 \\ \mathrm{C} & 4.17181700 & -1.37452200 & 0.63644400 \\ \mathrm{C} & 4.48814700 & -0.15997300 & 0.12908900 \\ \mathrm{O} & 3.34260000 & 0.47301400 & -0.27968500 \\ \mathrm{H} & 2.12398800 & -2.34445200 & 0.85272300 \\ \mathrm{H} & 4.87111100 & -2.09443000 & 1.03024400 \\ \mathrm{C} & -0.61009600 & 1.62479000 & -0.18021500 \\ \mathrm{C} & -0.09005800 & -0.61806800 & -0.23009200 \\ \mathrm{~N} & -1.11775300 & 0.32538300 & -0.13681000 \\ \mathrm{~N} & 1.07066600 & 0.12527600 & -0.40726300 \\ \mathrm{O} & -0.17150600 & -1.81268200 & -0.15422800 \\ \mathrm{O} & -1.21592200 & 2.65817300 & -0.21933000 \\ \mathrm{~N} & 0.77904800 & 1.48690400 & -0.16678800 \\ \mathrm{H} & 1.27173300 & 2.12063900 & -0.78669400 \\ \mathrm{C} & -2.50129300 & 0.00040400 & -0.02033400 \\ \mathrm{C} & -3.01473300 & -1.08873500 & -0.71807600 \\ \mathrm{C} & -3.31876900 & 0.78094000 & 0.79233700 \\ \mathrm{C} & -4.36285200 & -1.39945200 & -0.59009300 \\ \mathrm{H} & -2.36443600 & -1.69162800 & -1.33737000 \\ \mathrm{C} & -4.66733000 & 0.46582000 & 0.89867700 \\ \mathrm{H} & -2.90470400 & 1.63076800 & 1.31804300 \\ \mathrm{C} & -5.19232200 & -0.62443500 & 0.21322400 \\ \mathrm{H} & -4.76456400 & -2.25086800 & -1.12579100\end{array}$




$\begin{array}{lrrr}\mathrm{H} & -5.30697900 & 1.07583100 & 1.52494900 \\ \mathrm{H} & -6.24364100 & -0.86872000 & 0.30431600 \\ \mathrm{C} & 5.75606400 & 0.59076900 & -0.04753500 \\ \mathrm{H} & 5.74334500 & 1.52067100 & 0.52581400 \\ \mathrm{H} & 6.58819800 & -0.01989100 & 0.30105300 \\ \mathrm{H} & 5.92337400 & 0.83979200 & -1.09783700\end{array}$

\section{Reaction coordinates for Path-ES2}

Coordinate of $2 M F$

$\begin{array}{lrrr}\mathrm{C} & 0.63451500 & 0.10590400 & 0.00000600 \\ \mathrm{C} & -0.21874000 & 1.16153600 & 0.00000500 \\ \mathrm{C} & -1.54537500 & 0.61151300 & -0.00014400 \\ \mathrm{C} & -1.38926300 & -0.73240300 & 0.00008600 \\ \mathrm{O} & -0.07111800 & -1.05414800 & 0.00001500 \\ \mathrm{H} & 0.06147300 & 2.20257700 & 0.00000100 \\ \mathrm{H} & -2.48036800 & 1.14706800 & -0.00026100 \\ \mathrm{H} & -2.07773200 & -1.55999300 & 0.00015200 \\ \mathrm{C} & 2.11527400 & -0.01628300 & 0.00002700 \\ \mathrm{H} & 2.46101100 & -0.55701900 & 0.88409400 \\ \mathrm{H} & 2.46103400 & -0.55707900 & -0.88399400 \\ \mathrm{H} & 2.56506000 & 0.97602900 & 0.00000000\end{array}$

Coordinate of PhTAD

$\begin{array}{lrrr}\mathrm{C} & 1.56924400 & 1.09597100 & 0.24685500 \\ \mathrm{C} & 1.56921600 & -1.09573200 & -0.24781900 \\ \mathrm{~N} & 0.76011300 & 0.00009200 & -0.00031600 \\ \mathrm{~N} & 2.97785400 & 0.59785400 & 0.13660900 \\ \mathrm{~N} & 2.97783900 & -0.59803500 & -0.13586100 \\ \mathrm{O} & 1.28209600 & 2.21688800 & 0.49569500 \\ \mathrm{O} & 1.28203100 & -2.21688300 & -0.49556000 \\ \mathrm{C} & -0.66859100 & 0.00004800 & -0.00012000 \\ \mathrm{C} & -1.34952000 & -1.07231400 & 0.56575200 \\ \mathrm{C} & -1.34974600 & 1.07234700 & -0.56584000 \\ \mathrm{C} & -2.73869000 & -1.06763300 & 0.55663700 \\ \mathrm{H} & -0.80146200 & -1.89932100 & 0.99774500 \\ \mathrm{C} & -2.73891300 & 1.06753000 & -0.55641200 \\ \mathrm{H} & -0.80186600 & 1.89940200 & -0.99796500 \\ \mathrm{C} & -3.43490500 & -0.00008300 & 0.00019300 \\ \mathrm{H} & -3.27532000 & -1.90142900 & 0.99213200 \\ \mathrm{H} & -3.27571900 & 1.90127200 & -0.99179200 \\ \mathrm{H} & -4.51785800 & -0.00013300 & 0.00031600\end{array}$


Coordinate of $\mathrm{TS}_{1}$

$\begin{array}{lrrr}\mathrm{C} & -1.99444400 & -1.23533000 & -0.76987200 \\ \mathrm{C} & -2.56741600 & -1.37312900 & 0.53524100 \\ \mathrm{C} & -3.71847900 & -0.46314000 & 0.49460400 \\ \mathrm{C} & -3.54622300 & 0.31064800 & -0.58347200 \\ \mathrm{O} & -2.47690400 & -0.15695600 & -1.35674800 \\ \mathrm{H} & -1.22229900 & -1.79398400 & -1.27272800 \\ \mathrm{H} & -2.53738800 & -2.30019400 & 1.08801000 \\ \mathrm{H} & -4.44249400 & -0.31400300 & 1.27697000 \\ \mathrm{C} & -4.19564300 & 1.53523600 & -1.09454400 \\ \mathrm{H} & -3.48628000 & 2.36414000 & -1.03721500 \\ \mathrm{H} & -4.50208300 & 1.40806500 & -2.13431700 \\ \mathrm{H} & -5.06535600 & 1.77141100 & -0.48415400 \\ \mathrm{~N} & -1.31212500 & 0.82955500 & 0.98132000 \\ \mathrm{~N} & -1.27230000 & -0.48212400 & 1.09819800 \\ \mathrm{~N} & 0.76979600 & 0.06981900 & 0.43598400 \\ \mathrm{C} & 2.14445000 & 0.06121500 & 0.06784100 \\ \mathrm{C} & 2.65070500 & -0.99217600 & -0.69070600 \\ \mathrm{C} & 2.97201700 & 1.10454400 & 0.47723400 \\ \mathrm{C} & 3.99622500 & -0.99838300 & -1.03725500 \\ \mathrm{H} & 2.00231700 & -1.80487900 & -0.98914200 \\ \mathrm{C} & 4.31269600 & 1.08909500 & 0.11351900 \\ \mathrm{H} & 2.56178000 & 1.92094700 & 1.05472300 \\ \mathrm{C} & 4.83016500 & 0.04101500 & -0.64043000 \\ \mathrm{H} & 4.39052700 & -1.82056200 & -1.62204400 \\ \mathrm{H} & 4.95552700 & 1.90275300 & 0.42678900 \\ \mathrm{H} & 5.87758300 & 0.03402600 & -0.91637100 \\ \mathrm{C} & 0.02684800 & -1.03417600 & 0.73446300 \\ \mathrm{C} & -0.06730800 & 1.23956300 & 0.54941000 \\ \mathrm{O} & 0.30339200 & -2.20457400 & 0.76540700 \\ \mathrm{O} & 0.31214000 & 2.36173400 & 0.34943700\end{array}$

Coordinate of IM $_{1}$

\begin{tabular}{lrrr}
\hline $\mathrm{C}$ & -2.29718300 & -1.21678400 & -0.47085500 \\
$\mathrm{C}$ & -2.29630000 & -1.01172100 & 1.00261300 \\
$\mathrm{C}$ & -3.45630000 & -0.13711400 & 1.21959200 \\
$\mathrm{C}$ & -3.97388900 & 0.15054400 & 0.01914300 \\
$\mathrm{O}$ & -3.33974700 & -0.51876000 & -1.00991300 \\
$\mathrm{H}$ & -1.94073200 & -2.07549500 & -1.02189900 \\
$\mathrm{H}$ & -1.87593300 & -1.71775100 & 1.70556000 \\
$\mathrm{H}$ & -3.77871300 & 0.26192300 & 2.16573600
\end{tabular}




$\begin{array}{lrrr}\mathrm{C} & -5.04356700 & 1.07417800 & -0.41459900 \\ \mathrm{H} & -4.59160400 & 1.89715400 & -0.97312500 \\ \mathrm{H} & -5.74833300 & 0.55899500 & -1.06957000 \\ \mathrm{H} & -5.56889700 & 1.47445000 & 0.45026400 \\ \mathrm{~N} & -1.20631500 & 1.03669600 & -0.11360000 \\ \mathrm{~N} & -1.23305200 & -0.34440100 & 0.12637100 \\ \mathrm{~N} & 0.91217500 & 0.11690400 & 0.00936700 \\ \mathrm{C} & 2.33413600 & 0.06013300 & -0.01277000 \\ \mathrm{C} & 2.97303900 & -1.04210100 & -0.57630300 \\ \mathrm{C} & 3.06896100 & 1.10883900 & 0.53541500 \\ \mathrm{C} & 4.36132400 & -1.09489200 & -0.58258900 \\ \mathrm{H} & 2.38892500 & -1.85176500 & -0.99332300 \\ \mathrm{C} & 4.45651200 & 1.04524700 & 0.51198800 \\ \mathrm{H} & 2.55513500 & 1.96423000 & 0.95029700 \\ \mathrm{C} & 5.10650600 & -0.05268600 & -0.04210700 \\ \mathrm{H} & 4.85898900 & -1.95361500 & -1.01637500 \\ \mathrm{H} & 5.03105000 & 1.86189300 & 0.93180500 \\ \mathrm{H} & 6.18877600 & -0.09536400 & -0.05352500 \\ \mathrm{C} & 0.11657300 & -0.93849200 & 0.18723100 \\ \mathrm{C} & 0.09070000 & 1.35254600 & -0.19452900 \\ \mathrm{O} & 0.28174900 & -2.11621500 & 0.36323400 \\ \mathrm{O} & 0.62407600 & 2.41307100 & -0.39384500\end{array}$

Coordinate of $\mathrm{TS}_{2}$

\begin{tabular}{lrrr}
\hline $\mathrm{C}$ & -2.46994000 & 0.36075700 & 1.28242600 \\
$\mathrm{C}$ & -2.23238700 & 1.14442100 & 0.04292700 \\
$\mathrm{C}$ & -3.49859200 & 0.83607300 & -0.70661400 \\
$\mathrm{C}$ & -4.22140200 & -0.02544500 & -0.01287200 \\
$\mathrm{O}$ & -3.52157400 & -0.32590900 & 1.23637900 \\
$\mathrm{H}$ & -1.79294500 & 0.21836900 & 2.11983700 \\
$\mathrm{H}$ & -2.01047600 & 2.20047500 & 0.22891700 \\
$\mathrm{H}$ & -3.73674700 & 1.21792100 & -1.68611600 \\
$\mathrm{C}$ & -5.46929100 & -0.79312000 & -0.18085900 \\
$\mathrm{H}$ & -5.24448800 & -1.86179400 & -0.18847700 \\
$\mathrm{H}$ & -6.17007000 & -0.58639900 & 0.63003000 \\
$\mathrm{H}$ & -5.92848600 & -0.52212200 & -1.13004000 \\
$\mathrm{~N}$ & -1.09750000 & -0.87671900 & -0.64814100 \\
$\mathrm{~N}$ & -1.05837700 & 0.53570900 & -0.52856800 \\
$\mathrm{~N}$ & 1.00045400 & -0.07634700 & -0.24507000 \\
$\mathrm{C}$ & 2.40666200 & -0.05562500 & -0.06815500 \\
$\mathrm{C}$ & 3.13013000 & 1.08296600 & -0.42612500 \\
$\mathrm{C}$ & 3.06162000 & -1.16653000 & 0.46486200 \\
$\mathrm{C}$ & 4.50649000 & 1.10899600 & -0.24155300 \\
$\mathrm{H}$ & 2.61254600 & 1.94222300 & -0.83066500 \\
$\mathrm{C}$ & 4.44059800 & -1.12709100 & 0.63389100
\end{tabular}




$\begin{array}{lrrr}\mathrm{H} & 2.49278500 & -2.05029500 & 0.71450400 \\ \mathrm{C} & 5.16871400 & 0.00571000 & 0.28651400 \\ \mathrm{H} & 5.06210900 & 1.99740500 & -0.51743800 \\ \mathrm{H} & 4.94702200 & -1.99386200 & 1.04186400 \\ \mathrm{H} & 6.24307900 & 0.02825100 & 0.42411100 \\ \mathrm{C} & 0.19482300 & 1.01478300 & -0.13307900 \\ \mathrm{C} & 0.17459000 & -1.26848300 & -0.53587600 \\ \mathrm{O} & 0.41547600 & 2.15599400 & 0.23340200 \\ \mathrm{O} & 0.66379300 & -2.37672800 & -0.63065500\end{array}$

Coordinate of $\mathrm{IM}_{2}$

\begin{tabular}{lrrr}
\hline $\mathrm{C}$ & -2.15545000 & -0.71836000 & 0.60065600 \\
$\mathrm{C}$ & -2.16729800 & 0.82816100 & 0.78084900 \\
$\mathrm{C}$ & -3.53486900 & 1.16489200 & 0.28572500 \\
$\mathrm{C}$ & -4.16164600 & 0.03588600 & -0.05014900 \\
$\mathrm{O}$ & -3.42928600 & -1.11034500 & 0.18246100 \\
$\mathrm{H}$ & -1.80209000 & -1.39729200 & 1.37524100 \\
$\mathrm{H}$ & -1.85466800 & 1.28526100 & 1.71977300 \\
$\mathrm{H}$ & -3.94100900 & 2.15962300 & 0.20025900 \\
$\mathrm{C}$ & -5.51270000 & -0.20990700 & -0.60888600 \\
$\mathrm{H}$ & -5.42410800 & -0.70602000 & -1.57773800 \\
$\mathrm{H}$ & -6.07714400 & -0.86871200 & 0.05438200 \\
$\mathrm{H}$ & -6.04920700 & 0.72917700 & -0.73162800 \\
$\mathrm{~N}$ & -1.17874900 & -0.52900300 & -0.49847100 \\
$\mathrm{~N}$ & -1.09670000 & 0.89677600 & -0.26668800 \\
$\mathrm{~N}$ & 0.96182600 & 0.03667600 & -0.18874800 \\
$\mathrm{C}$ & 2.38460600 & -0.02620500 & -0.10878800 \\
$\mathrm{C}$ & 3.12952000 & 1.02005000 & -0.64651200 \\
$\mathrm{C}$ & 3.01022500 & -1.11147300 & 0.49805600 \\
$\mathrm{C}$ & 4.51485900 & 0.97861400 & -0.56950800 \\
$\mathrm{H}$ & 2.62552700 & 1.85993300 & -1.10675000 \\
$\mathrm{C}$ & 4.39901200 & -1.14454100 & 0.55603000 \\
$\mathrm{H}$ & 2.41974200 & -1.92116100 & 0.90282500 \\
$\mathrm{C}$ & 5.15348600 & -0.10363300 & 0.02801400 \\
$\mathrm{H}$ & 5.09541000 & 1.79430700 & -0.98273800 \\
$\mathrm{H}$ & 4.88950000 & -1.99038700 & 1.02222600 \\
$\mathrm{H}$ & 6.23493300 & -0.13429900 & 0.08145100 \\
$\mathrm{C}$ & 0.21994400 & 1.20210200 & 0.08041600 \\
$\mathrm{C}$ & 0.11322800 & -1.06385100 & -0.35355300 \\
$\mathrm{O}$ & 0.62404000 & 2.24387000 & 0.51199500 \\
$\mathrm{O}$ & 0.39536700 & -2.22752200 & -0.34871400
\end{tabular}

Coordinate of $\mathrm{TS}_{3}$ 


$\begin{array}{lrrr}\mathrm{C} & -2.19916100 & -0.86826900 & 0.27633000 \\ \mathrm{C} & -2.62399700 & 0.10278100 & 1.35017800 \\ \mathrm{C} & -3.89724000 & 0.51994700 & 1.10596700 \\ \mathrm{C} & -4.26734200 & -0.04009500 & -0.13571900 \\ \mathrm{O} & -3.38328100 & -0.83055600 & -0.64314300 \\ \mathrm{H} & -2.08543600 & -1.92227700 & 0.54489900 \\ \mathrm{H} & -2.00756500 & 0.29637400 & 2.21562300 \\ \mathrm{H} & -4.49887000 & 1.21348500 & 1.67031800 \\ \mathrm{C} & -5.50276700 & 0.19412100 & -0.91314900 \\ \mathrm{H} & -5.24533800 & 0.83361000 & -1.76343200 \\ \mathrm{H} & -5.88057900 & -0.74888300 & -1.31018300 \\ \mathrm{H} & -6.25860900 & 0.68969800 & -0.30818600 \\ \mathrm{~N} & -1.08215300 & -0.32871400 & -0.33362000 \\ \mathrm{~N} & -1.03680700 & 1.06645300 & -0.06205600 \\ \mathrm{~N} & 1.02988800 & 0.11304600 & -0.09081300 \\ \mathrm{C} & 2.44649600 & 0.00953600 & -0.06688800 \\ \mathrm{C} & 3.21831700 & 1.04971400 & -0.58388700 \\ \mathrm{C} & 3.05784500 & -1.12627200 & 0.46308800 \\ \mathrm{C} & 4.60311000 & 0.94726900 & -0.56251000 \\ \mathrm{H} & 2.73245700 & 1.93180800 & -0.97603600 \\ \mathrm{C} & 4.44490100 & -1.21788400 & 0.46641500 \\ \mathrm{H} & 2.45131400 & -1.93283700 & 0.85004600 \\ \mathrm{C} & 5.22283100 & -0.18418500 & -0.04171400 \\ \mathrm{H} & 5.19999000 & 1.75968600 & -0.95954200 \\ \mathrm{H} & 4.91566700 & -2.10480900 & 0.87355500 \\ \mathrm{H} & 6.30358000 & -0.25905900 & -0.03236700 \\ \mathrm{C} & 0.27877600 & 1.34885000 & 0.08935300 \\ \mathrm{C} & 0.16861800 & -0.94396600 & -0.24159500 \\ \mathrm{O} & 0.80604900 & 2.41074100 & 0.33428900 \\ \mathrm{O} & 0.36405200 & -2.13788000 & -0.27045700\end{array}$

Coordinate of $\mathrm{IM}_{3}$

$\begin{array}{lrrr}\mathrm{C} & 2.10650800 & 0.37503900 & -0.02960800 \\ \mathrm{C} & 2.68238600 & -0.93367700 & 0.14906200 \\ \mathrm{C} & 4.00907000 & -1.17200900 & 0.18460200 \\ \mathrm{C} & 5.11171900 & -0.17438800 & 0.04428700 \\ \mathrm{O} & 4.90963600 & 1.00456800 & -0.13911400 \\ \mathrm{H} & 2.00815100 & -1.76974400 & 0.26564000 \\ \mathrm{H} & 4.32475200 & -2.20007000 & 0.33183200 \\ \mathrm{C} & -0.99868500 & 1.79010400 & -0.23119100 \\ \mathrm{C} & -0.28584100 & -0.38027000 & 0.03744800 \\ \mathrm{~N} & -1.37900800 & 0.38969800 & -0.05174100 \\ \mathrm{~N} & 0.82396600 & 0.62911800 & -0.07627600 \\ \mathrm{O} & -0.12752100 & -1.55683200 & 0.17052500 \\ \mathrm{O} & -1.80864800 & 2.66453000 & -0.35044200\end{array}$




$\begin{array}{lrrc}\mathrm{N} & 0.35793200 & 1.87213300 & -0.23300000 \\ \mathrm{H} & 2.73699900 & 1.24862800 & -0.13326900 \\ \mathrm{C} & -2.71708400 & -0.09895000 & -0.00111400 \\ \mathrm{C} & -3.67688000 & 0.61670300 & 0.70827900 \\ \mathrm{C} & -3.04147600 & -1.28264700 & -0.65695900 \\ \mathrm{C} & -4.97881600 & 0.13426600 & 0.75420000 \\ \mathrm{H} & -3.40673100 & 1.54168400 & 1.19885800 \\ \mathrm{C} & -4.34523700 & -1.75788500 & -0.59278600 \\ \mathrm{H} & -2.28051400 & -1.82655500 & -1.20135200 \\ \mathrm{C} & -5.31555500 & -1.05141500 & 0.10958200 \\ \mathrm{H} & -5.73200600 & 0.68944400 & 1.29974300 \\ \mathrm{H} & -4.60124600 & -2.68051100 & -1.09896800 \\ \mathrm{H} & -6.33203600 & -1.42305600 & 0.15338600 \\ \mathrm{C} & 6.50684000 & -0.73913900 & 0.14727900 \\ \mathrm{H} & 7.23633900 & 0.05770800 & 0.02330000 \\ \mathrm{H} & 6.64094700 & -1.22095800 & 1.11973200 \\ \mathrm{H} & 6.65569500 & -1.50575800 & -0.61801400\end{array}$

Coordinate of $\mathrm{TS}_{4}$

$\begin{array}{lrrr}\mathrm{C} & -2.23275200 & 0.21521300 & 0.12696500 \\ \mathrm{C} & -2.47323900 & -0.88160500 & 1.00895600 \\ \mathrm{C} & -3.81531700 & -1.18310500 & 0.95259800 \\ \mathrm{C} & -4.35772600 & -0.35344000 & -0.03727700 \\ \mathrm{O} & -3.44934900 & 0.41334200 & -0.57511200 \\ \mathrm{H} & -1.71091300 & -1.32238600 & 1.63531300 \\ \mathrm{H} & -4.36652400 & -1.90308100 & 1.53526100 \\ \mathrm{C} & 0.63491100 & 1.69458000 & 0.31080400 \\ \mathrm{C} & 0.01153300 & -0.38552800 & -0.51392500 \\ \mathrm{~N} & 1.08191600 & 0.38024300 & -0.12804600 \\ \mathrm{~N} & -1.06071000 & 0.52003700 & -0.57149800 \\ \mathrm{O} & -0.07250700 & -1.57177900 & -0.73133200 \\ \mathrm{O} & 1.36354700 & 2.49953800 & 0.83741700 \\ \mathrm{~N} & -0.68656500 & 1.79537600 & 0.01439000 \\ \mathrm{H} & -1.96097200 & 1.28557900 & 0.78287600 \\ \mathrm{C} & 2.43096800 & -0.06571100 & -0.07946400 \\ \mathrm{C} & 3.45468600 & 0.84185900 & -0.34993700 \\ \mathrm{C} & 2.72600400 & -1.39348800 & 0.22707800 \\ \mathrm{C} & 4.77468200 & 0.41395400 & -0.30612000 \\ \mathrm{H} & 3.21208000 & 1.87200500 & -0.57062500 \\ \mathrm{C} & 4.05278900 & -1.80810400 & 0.25669500 \\ \mathrm{H} & 1.92532300 & -2.09348400 & 0.41943900 \\ \mathrm{C} & 5.08038700 & -0.90976800 & -0.00570100 \\ \mathrm{H} & 5.56813600 & 1.12269800 & -0.51086200 \\ \mathrm{H} & 4.27954600 & -2.84201600 & 0.48893000 \\ \mathrm{H} & 6.11237300 & -1.23836600 & 0.02255100 \\ \mathrm{C} & -5.75639900 & -0.19436400 & -0.49499200\end{array}$




$\begin{array}{lrrr}H & -6.36853500 & -1.02242600 & -0.14300500 \\ H & -6.16289800 & 0.74156800 & -0.10166600 \\ H & -5.78679600 & -0.14144400 & -1.58400000\end{array}$

Coordinate of Product

$\begin{array}{lrrr}\mathrm{C} & 2.31667700 & -0.35650800 & 0.00380300 \\ \mathrm{C} & 2.74099200 & -1.51247600 & 0.56152500 \\ \mathrm{C} & 4.17181700 & -1.37452200 & 0.63644400 \\ \mathrm{C} & 4.48814700 & -0.15997300 & 0.12908900 \\ \mathrm{O} & 3.34260000 & 0.47301400 & -0.27968500 \\ \mathrm{H} & 2.12398800 & -2.34445200 & 0.85272300 \\ \mathrm{H} & 4.87111100 & -2.09443000 & 1.03024400 \\ \mathrm{C} & -0.61009600 & 1.62479000 & -0.18021500 \\ \mathrm{C} & -0.09005800 & -0.61806800 & -0.23009200 \\ \mathrm{~N} & -1.11775300 & 0.32538300 & -0.13681000 \\ \mathrm{~N} & 1.07066600 & 0.12527600 & -0.40726300 \\ \mathrm{O} & -0.17150600 & -1.81268200 & -0.15422800 \\ \mathrm{O} & -1.21592200 & 2.65817300 & -0.21933000 \\ \mathrm{~N} & 0.77904800 & 1.48690400 & -0.16678800 \\ \mathrm{H} & 1.27173300 & 2.12063900 & -0.78669400 \\ \mathrm{C} & -2.50129300 & 0.00040400 & -0.02033400 \\ \mathrm{C} & -3.01473300 & -1.08873500 & -0.71807600 \\ \mathrm{C} & -3.31876900 & 0.78094000 & 0.79233700 \\ \mathrm{C} & -4.36285200 & -1.39945200 & -0.59009300 \\ \mathrm{H} & -2.36443600 & -1.69162800 & -1.33737000 \\ \mathrm{C} & -4.66733000 & 0.46582000 & 0.89867700 \\ \mathrm{H} & -2.90470400 & 1.63076800 & 1.31804300 \\ \mathrm{C} & -5.19232200 & -0.62443500 & 0.21322400 \\ \mathrm{H} & -4.76456400 & -2.25086800 & -1.12579100 \\ \mathrm{H} & -5.30697900 & 1.07583100 & 1.52494900 \\ \mathrm{H} & -6.24364100 & -0.86872000 & 0.30431600 \\ \mathrm{C} & 5.75606400 & 0.59076900 & -0.04753500 \\ \mathrm{H} & 5.74334500 & 1.52067100 & 0.52581400 \\ \mathrm{H} & 6.58819800 & -0.01989100 & 0.30105300 \\ \mathrm{H} & 5.92337400 & 0.83979200 & -1.09783700\end{array}$




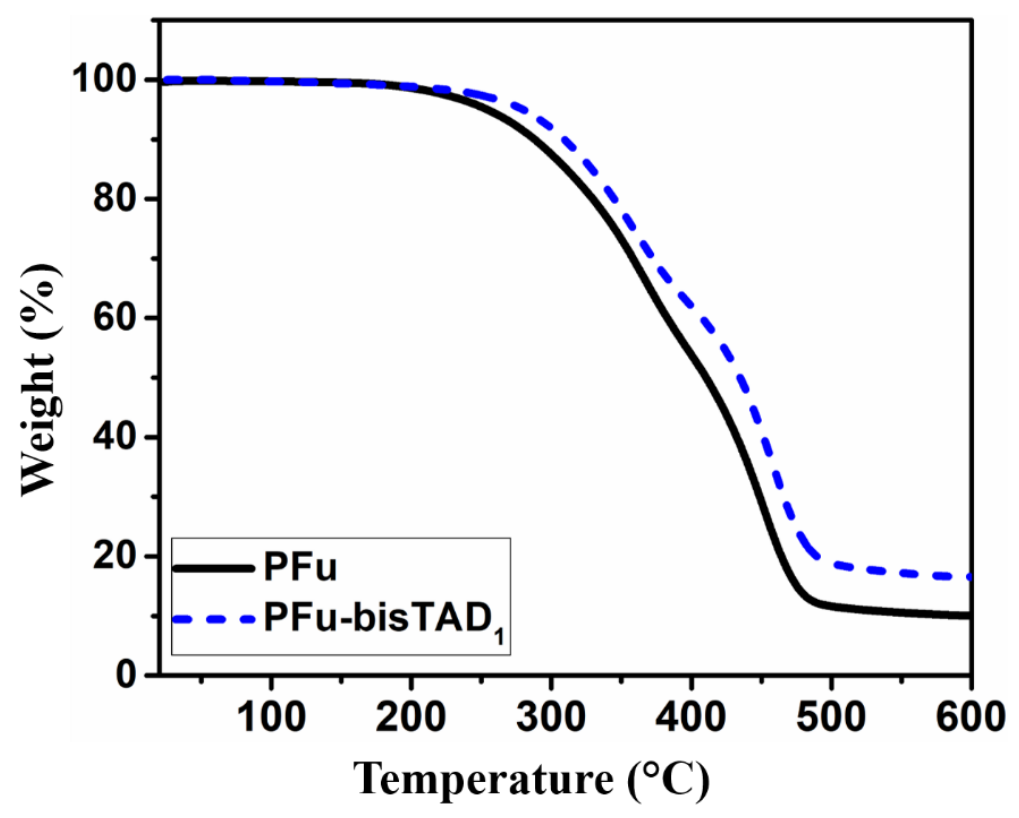

Figure S11. TGA analysis of PFu and PFu-bisTAD polymers.

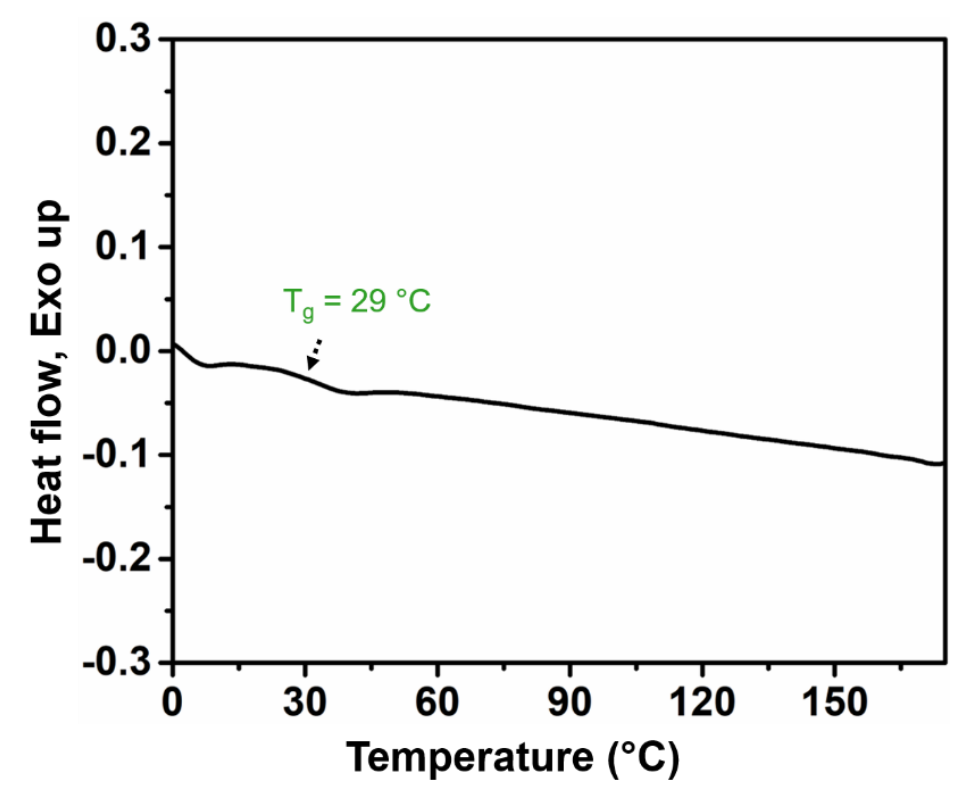

Figure S12. DSC analysis of $1^{\text {st }}$ heating curve of PFu. 


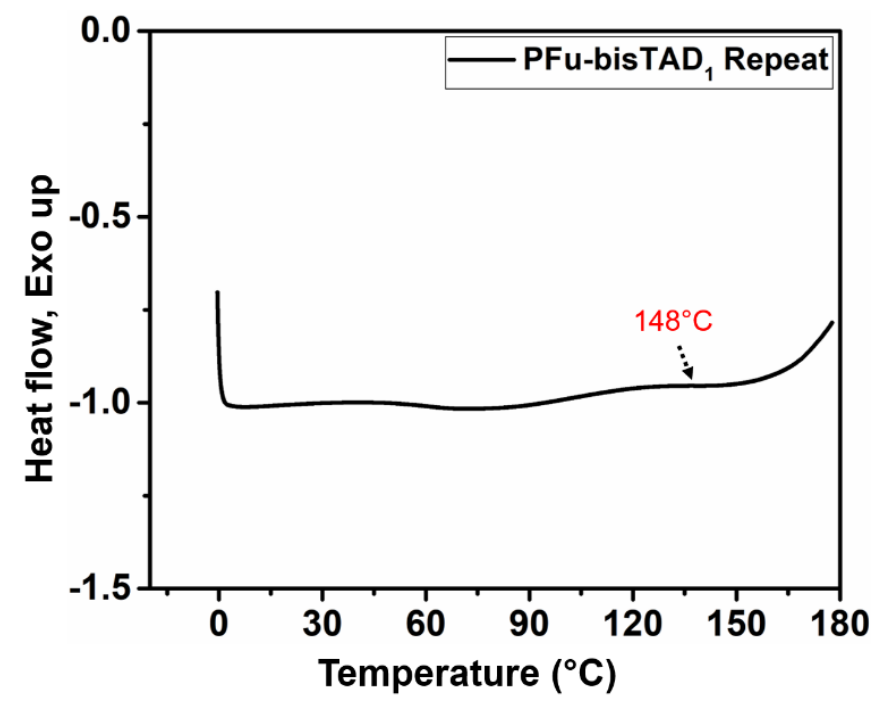

Figure S13. Repeat DSC analysis ( $2^{\text {nd }}$ DSC cycle) of $1^{\text {st }}$ heating curve of PFu-bisTAD chemical network.

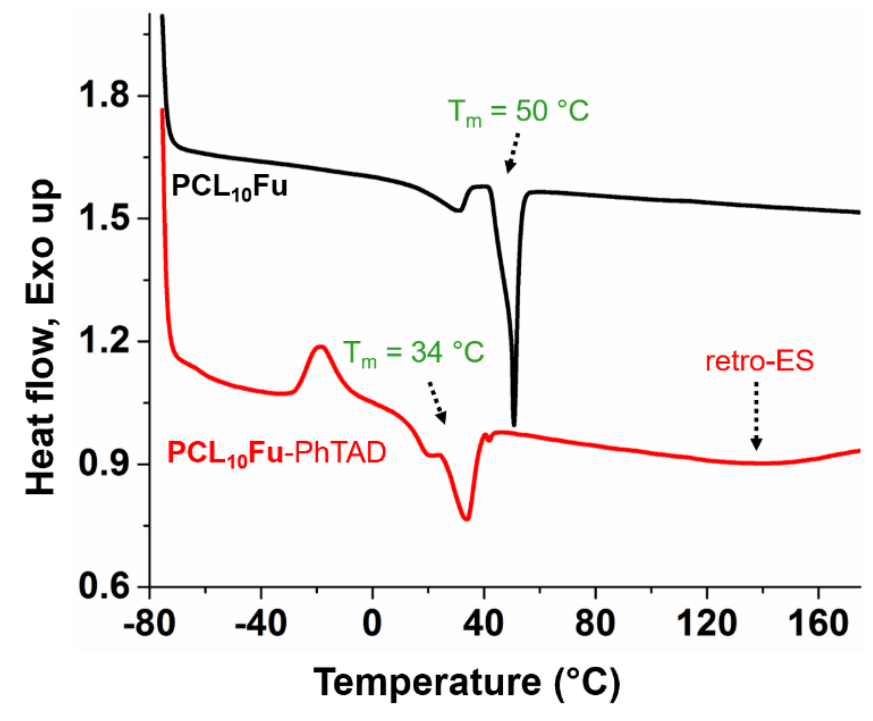

Figure S14. DSC analysis of $1^{\text {st }}$ heating curve of PCL10Fu and PCL $\mathbf{P 0 F u}-\mathrm{PhTAD}$. 

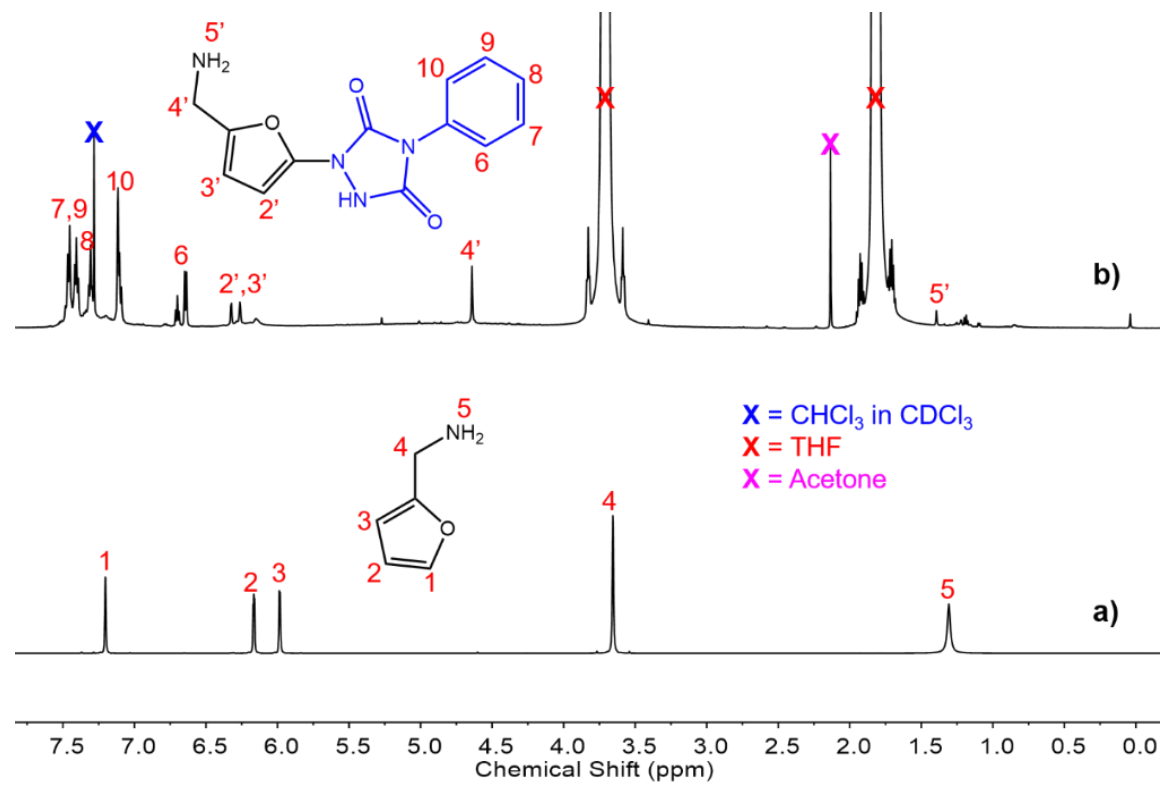

Figure S15. ${ }^{1} \mathrm{H}$ NMR spectrum of a) FA and b) FA-PhTAD in $\mathrm{CDCl}_{3}$ solvent.

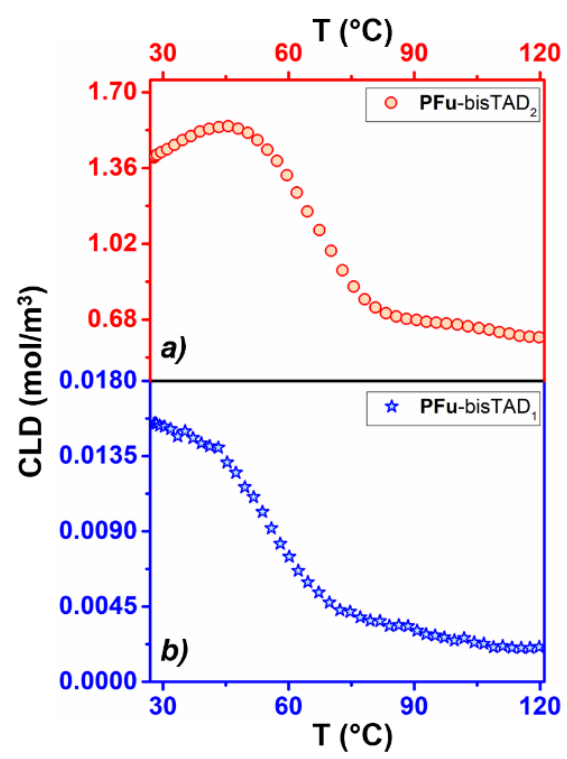

Figure S16. Plots of crosslink density $(C L D)$ of a) PFu-bisTAD $_{1}$ and b) $\mathbf{P F u}$-bisTAD ${ }_{2}$ vs. temperature (T). CLD was calculated according the equation S1. 

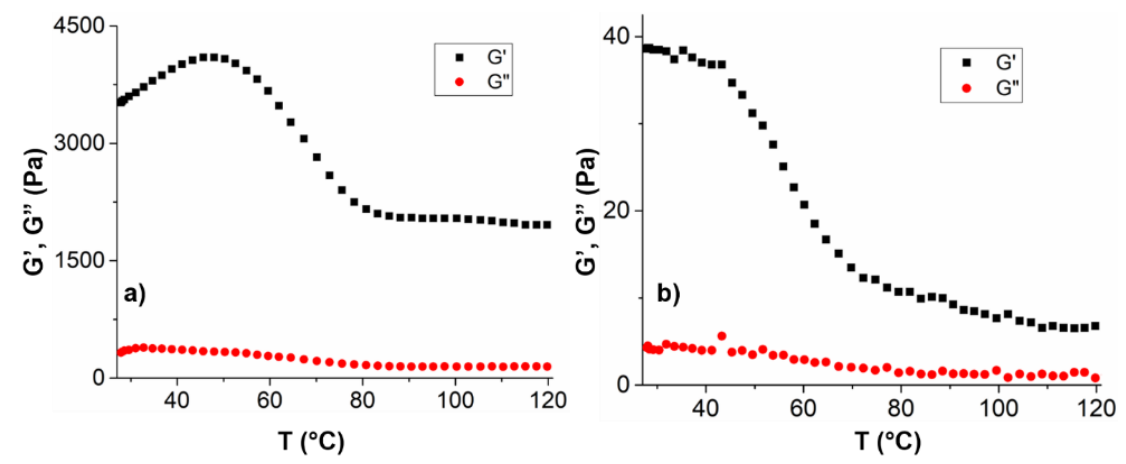

Figure S17. Temperature sweep of a) $\mathbf{P F u}-$ bisTAD $_{2}$ and b) PFu-bisTAD 1 to manifest the crossover of $G^{\prime}$ and $G^{\prime \prime}$.

Table S3. Summary of healing efficacy $\left(\boldsymbol{E}_{\boldsymbol{H}}\right)$ of PFu-bisTAD 1

\begin{tabular}{lcccc}
\hline Crosslinked Polymers $^{\mathrm{a}}$ & $\begin{array}{c}\text { Post-notch } \\
\text { Depth }(\mu \mathrm{m})\end{array}$ & $\begin{array}{c}\text { Post-heal Depth } \\
(\mu \mathrm{m})\end{array}$ & $E_{H}(\%)^{\mathrm{b}}$ & Duration of \\
& & & & \\
\hline PFu-bisTAD 1 network & 30.3 & 5.1 & $83.3 \pm 1.6$ & $76 \mathrm{~h}$ \\
\hline
\end{tabular}

${ }^{\mathrm{a}}$ The initial depth of all the polymer samples was $\sim 0.0 \mu \mathrm{m}$.
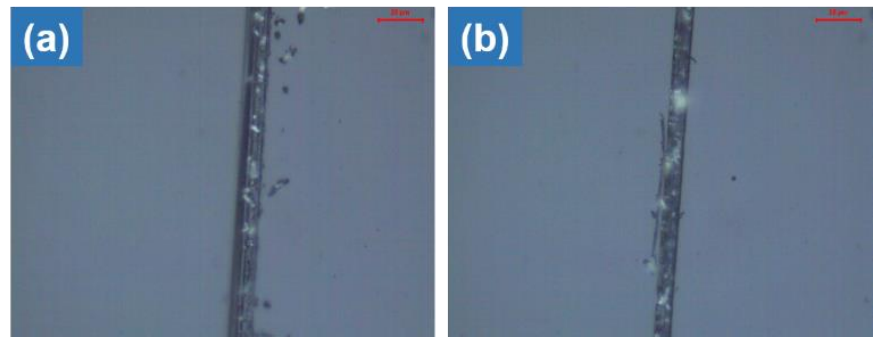

Figure S18. Optical microscopy images of (a) notched PFu at r.t., and (b) after heating at $130{ }^{\circ} \mathrm{C}$ for $76 \mathrm{~h}$ followed by cooling at r.t. 


\section{References:}

1. Mondal, P.; Raut, S. K.; Singha, N. K. Thermally amendable tailor-made acrylate copolymers via RAFT polymerization and ultrafast alder-ene "click" chemistry. J. Polym. Sci. Part A: Polym. Chem. 2018, 56, 2310-2318.

2. Solvay Advanced Polymers, Solution processing guide for polymer membranes. Tech. Bull., 2010, 1-11.

3. Zheng, W.; Wu, X.; Huang, Y. Impact of polymer addition, electrolyte, clay and antioxidant on rheological properties of polymer fluid at high temperature and high pressure. $J$. Petrol. Explor. Prod. Technol. 2020, 10 (2), 663-671.

4. Lv, Y.; Wu, J.; Zhang, J.; Niu, Y.; Liu, C.-Y.; He, J.; Zhang, J. Rheological properties of cellulose/ionic liquid/dimethylsulfoxide (DMSO) solutions. Polymer 2012, 53 (12), 2524-2531.

5. Zhang, L.; Shi, W.; Wang, J.; Jin, L.; Hu, G.; Zheng, Q.; Xie, H.; Chen, P. Unique gelation and rheological properties of the cellulose/CO2-based reversible ionic liquid/DMSO solutions. Carbohydrate polymers 2019, 222, 115024.

6. R. J. Young and P. A. Lovell, Introduction to polymers, CRC press, Taylor \& Francis Group, Boca Raton, Florida, Third edn., 2011.

7. Zhao, Y.; Truhlar, D. G. The M06 suite of density functionals for main group thermochemistry, thermochemical kinetics, noncovalent interactions, excited states, and transition elements: two new functionals and systematic testing of four M06-class functionals and 12 other functionals. Theor. Chem. Acc. 2008, 120 (1-3), 215-241.

8. Liu, L. P.; Malhotra, D.; Paton, R. S.; Houk, K.; Hammond, G. B. The [4+ 2], not [2+ 2], Mechanism Occurs in the Gold-Catalyzed Intramolecular Oxygen Transfer Reaction of 2-Alkynyl1, 5-Diketones. Angew. Chem. Int. Ed. 2010, 49 (48), 9132-9135. 
9. Krishnan, R.; Binkley, J. S.; Seeger, R.; Pople, J. A. Self-consistent molecular orbital methods. XX. A basis set for correlated wave functions. J. Chem. Phys. 1980, 72 (1), 650-654.

10. Clark, T.; Chandrasekhar, J.; Spitznagel, G. W.; Schleyer, P. V. R. Efficient diffuse function-augmented basis sets for anion calculations. III. The $3-21+\mathrm{G}$ basis set for first-row elements, Li-F. J. Comput. Chem. 1983, 4 (3), 294-301.

11. Frisch, M. J.; Trucks, G. W.; Schlegel, H. B.; Scuseria, G. E.; Robb, M. A.; Cheeseman, J. R.; Scalmani, G.; Barone, V.; Petersson, G. A.; Nakatsuji, H.; Li, X.; Caricato, M.; Marenich, A. V.; Bloino, J.; Janesko, B. G.; Gomperts, R.; Mennucci, B.; Hratchian, H. P.; Ortiz, J. V.; Izmaylov, A. F.; Sonnenberg, J. L.; Williams-Young, D.; Ding, F.; Lipparini, F.; Egidi, F.; Goings, J.; Peng, B.; Petrone, A.; Henderson, T.; Ranasinghe, D.; Zakrzewski, V. G.; Gao, J.; Rega, N.; Zheng, G.; Liang, W.; Hada, M.; Ehara, M.; Toyota, K.; Fukuda, R.; Hasegawa, J.; Ishida, M.; Nakajima, T.; Honda, Y.; Kitao, O.; Nakai, H.; Vreven, T.; Throssell, K.; Montgomery, J. A., Jr.; Peralta, J. E.; Ogliaro, F.; Bearpark, M. J.; Heyd, J. J.; Brothers, E. N.; Kudin, K. N.; Staroverov, V. N.; Keith, T. A.; Kobayashi, R.; Normand, J.; Raghavachari, K.; Rendell, A. P.; Burant, J. C.; Iyengar, S. S.; Tomasi, J.; Cossi, M.; Millam, J. M.; Klene, M.; Adamo, C.; Cammi, R.; Ochterski, J. W.; Martin, R. L.; Morokuma, K.; Farkas, O.; Foresman, J. B.; Fox, D. J., Gaussian 16, Revision C.01Gaussian, Inc., Wallingford CT, 2016. 